\title{
Impact of genome architecture on the functional activation and repression of Hox regulatory landscapes
}

Eddie Rodríguez-Carballo ${ }^{1}$, Lucille Lopez-Delisle ${ }^{2}$, Nayuta Yakushiji-Kaminatsuii ${ }^{2,5}$, Asier Ullate-Agote ${ }^{3}$ and Denis Duboule $1,2,4^{*}$

\begin{abstract}
Background: The spatial organization of the mammalian genome relies upon the formation of chromatin domains of various scales. At the level of gene regulation in cis, collections of enhancer sequences define large regulatory landscapes that usually match with the presence of topologically associating domains (TADs). These domains often contain ranges of enhancers displaying similar or related tissue specificity, suggesting that in some cases, such domains may act as coherent regulatory units, with a global on or off state. By using the HoxD gene cluster, which specifies the topology of the developing limbs via highly orchestrated regulation of gene expression, as a paradigm, we investigated how the arrangement of regulatory domains determines their activity and function.

Results: Proximal and distal cells in the developing limb express different levels of Hoxd genes, regulated by flanking 3' and 5' TADs, respectively. We characterized the effect of large genomic rearrangements affecting these two TADs, including their fusion into a single chromatin domain. We show that, within a single hybrid TAD, the activation of both proximal and distal limb enhancers globally occurred as when both TADs are intact. However, the activity of the 3' TAD in distal cells is generally increased in the fused TAD, when compared to wild type where it is silenced. Also, target gene activity in distal cells depends on whether or not these genes had previously responded to proximal enhancers, which determines the presence or absence of H3K27me3 marks. We also show that the polycomb repressive complex 2 is mainly recruited at the Hox gene cluster and can extend its coverage to far-cis regulatory sequences as long as confined to the neighboring TAD structure.
\end{abstract}

Conclusions: We conclude that antagonistic limb proximal and distal enhancers can exert their specific effects when positioned into the same TAD and in the absence of their genuine target genes. We also conclude that removing these target genes reduced the coverage of a regulatory landscape by chromatin marks associated with silencing, which correlates with its prolonged activity in time.

Keywords: Genome architecture, TAD, Polycomb, Tetrapod evolution, Enhancers, Regulatory landscapes

\section{Background}

Attempts to understand the spatial organization of the genome in the nucleus have recently led to models accounting for the relationship between genome structure and gene regulation (see [1]). The development of chromosome conformation capture techniques associated with deep

\footnotetext{
* Correspondence: Denis.Duboule@unige.ch

'Laboratory of Developmental Genomics, Department of Genetics and

Evolution, University of Geneva, 1211 Geneva 4, Switzerland

${ }^{2}$ School of Life Sciences, Federal Institute of Technology, Lausanne, 1015

Lausanne, Switzerland

Full list of author information is available at the end of the article
}

sequencing has thus allowed the resolution of DNA interactions at a small scale [2]. These interactions can be either structural or functional, i.e., they can be present regardless of the transcriptional outcome or alternatively, they can fluctuate according to cell type-specific context depending upon the transcriptional status [3]. Constitutive contacts generally tend to fit the loop extrusion model, whereby the packed network of chromatin loops would form as a result of DNA extrusion by an ATP-dependent cohesin-based complex. In this model, the loops are stabilized whenever

(c) The Author(s). 2019 Open Access This article is distributed under the terms of the Creative Commons Attribution 4.0 International License (http://creativecommons.org/licenses/by/4.0/), which permits unrestricted use, distribution, and reproduction in any medium, provided you give appropriate credit to the original author(s) and the source, provide a link to the Creative Commons license, and indicate if changes were made. The Creative Commons Public Domain Dedication waiver (http://creativecommons.org/publicdomain/zero/1.0/) applies to the data made available in this article, unless otherwise stated. 
this cohesin ring meets two CTCF molecules bound with convergent orientations [4-6].

Chromatin is organized in several levels of interactions, loops, and domains. At the level of gene regulation, topologically associating domains (TADs) [7-9] usually match large domains of long-range gene regulation referred to as regulatory landscapes [10]. These structures are globally detected in all cell types and conserved across vertebrate species [7, 11-15]. The experimental depletion of either CTCF or cohesin subunits leads to a loss of both loop organization and TAD structure. Under these conditions, however, the effects upon gene transcription were limited and the formation of larger structures (compartments), which may also be functionally relevant, still occurred although in an altered manner [16-20].

Compartments contain chromatin domains labeled by various epigenetic marks. Inactive chromatin domains labeled by histone $\mathrm{H} 3$ lysine 27 trimethylation (H3K27me3), resulting from the presence of polycomb group protein complexes, have been associated either with compartment A [21] or with a compartment B1, distinct from the genuine heterochromatin B compartment [5], which segregate from other chromatin domains, possibly through phase separation [22, 23]. In addition, facultative heterochromatin (H3K27me3-positive) was shown to correlate with long-distance interactions either in stem cells [24-26] or during embryonic development [21, 27].

Distinct functional states associated with various chromatin structures are not as clear when TADs are considered. While several examples exist showing the functional coherence of multiple enhancer sequences present within one particular TAD [28-32], the definition of TADs as global independent regulatory units still lacks experimental evidence. In many instances, indeed, TADs include either series of enhancers with the same-or related-specificity or enhancers with distinct tissue-specific potentials but involved in the pleiotropic regulation of the same target gene(s). However, whether or not the entire TAD adopts a global on or off state, for example, related to a particular architecture, remains to be established.

A useful experimental paradigm to address this question is the mammalian $\operatorname{HoxD}$ gene cluster, a group of genes located at the intersection between two TADs displaying distinct functional specificities [33]. During limb development, enhancers in the telomeric TAD (3'-TAD) regulate the transcription of Hoxd8 to Hoxd11 in proximal limb bud cells. Subsequently, enhancers in the centromeric TAD (5'TAD) control the Hoxd9 to Hoxd13 genes in distal limb bud cells [33]. These different sets of target genes responding to either one of the regulatory domains are determined by a robust boundary, centered around Hoxd11 and relying upon a collection of bound CTCF sites. Genetic analyses in vivo revealed that this boundary was very resilient and that even a full deletion of the gene cluster was unable to merge both TADs into one single domain, likely due to a few remaining occupied CTCF sites [34].

The analysis of different developmental contexts where Hoxd genes are transcribed demonstrates that these two TADs are functionally exclusive from one another, i.e., the concomitant function of enhancers belonging to the two domains has not been observed thus far. This is due in part to the fact that the main target gene responding to $5^{\prime}$-TAD enhancers is Hoxd13, whose product, along with that of Hoxa13, has a negative effect over 3 '-TAD enhancers through direct binding as observed in ChIPseq experiments [32, 35]. These TAD-exclusive regulations can also be followed by the appearance of relevant chromatin marks: while the 3 '-TAD is largely covered by H3K27ac marks in proximal limb bud cells, it becomes rapidly decorated by $\mathrm{H} 3 \mathrm{~K} 27 \mathrm{me} 3$ marks at the time the $5^{\prime}$-TAD starts to be active in distal cells and to accumulate H3K27ac marks [33]. Therefore, in distal cells, H3K27me3 marks not only decorate the Hoxd1 to Hoxd8 genes (they are no longer transcribed) but also large DNA intervals within the 3 '-TAD reflecting the off state of this regulatory landscape and re-enforcing the idea that it may behave as a coherent regulatory unit.

In this study, we challenged this hypothesis by investigating the effects of combining the two TADs into a single domain (a fused-TAD), after deletion of a large piece of DNA containing the HoxD cluster as well as other boundary elements. After merging, this fused-TAD contained enhancers that do not normally function in the same cellular context. We asked whether these various enhancers would keep their initial functional specificities or, alternatively, if they would all be active or repressed concomitantly, as a result of this new topological proximity. In addition, we used a set of engineered inversions, which disconnect the target Hoxd genes from their TADs to evaluate the functional and epigenetic behavior of these regulatory sequences in the absence of their target genes.

\section{Results}

In order to better visualize the spatial distribution of the two TADs associated with the HoxD cluster (Fig. 1a, b), we modeled their structures in $3 \mathrm{D}$ by using $\mathrm{Hi}-\mathrm{C}$ matrices [34] for both distal and proximal E12.5 limb bud cells (Fig. 1c) and the TADkit scripts package as a 3D modeling viewer [36]. In the wild-type condition, the HoxD cluster exerted a strong boundary effect and was thus positioned between the $3^{\prime}$-TAD and $5^{\prime}$-TAD regulatory domains, in both distal and proximal limb cells (Fig. 1c). In both tissues, a region called CS38-41 (Fig. 1, red disk) established a weaker boundary between two sub-TADs in the $3^{\prime}$-TAD. The structure and separation 

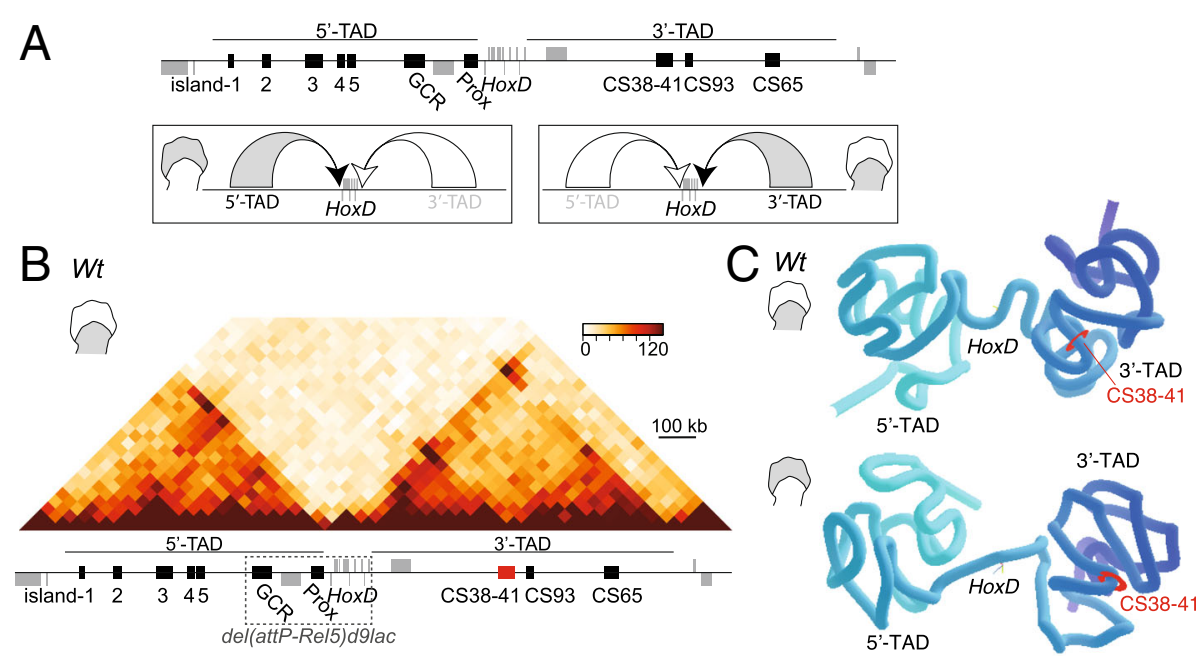

D del(attP-Rel5)d9lac

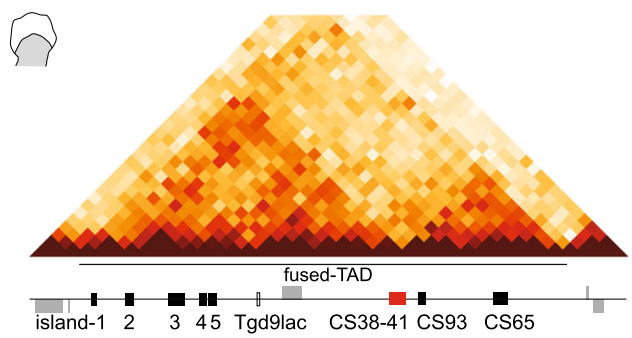

E del(attP-Re/5)d9lac

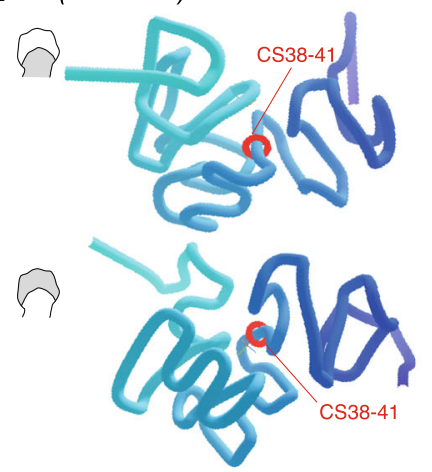

F
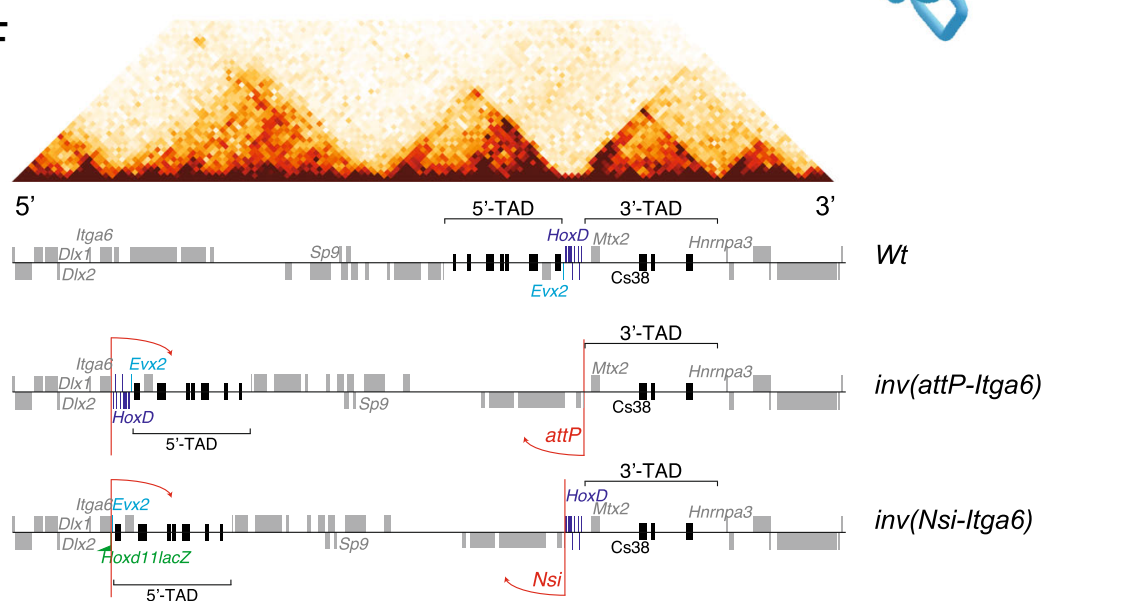

Fig. 1 (See legend on next page.) 
(See figure on previous page.)

Fig. $13 D$ representation of the HoxD locus in control $(W t)$ and mutant limb buds. a Representation of the HoxD cluster and their associated regulatory domains (5'-TAD and 3'-TAD). The schemes below show the differential activation by the 5'-TAD and the 3'-TAD, in distal and proximal limb bud cells, respectively. $\mathbf{b} \mathrm{Hi}-\mathrm{C}$ map showing the distribution of TADs on either side of the HoxD locus in proximal limb and its associated genes (gray boxes) and regulatory regions (black and red boxes). The dashed rectangle illustrates the deletion in the del(attP-Rel5)d9lac allele. $\mathbf{c}$ Three-dimensional modeling of HoxD-associated TADs derived from Hi-C datasets obtained from wild-type (Wt) proximal (top) and distal (bottom) limb bud cells (schemes on the left). $\mathbf{d}$ Hi-C map showing the extent of the fused-TAD upon deletion of the HoxD locus in proximal limb cells, as well as the remaining genes (gray boxes) and regulatory regions (black and red boxes). e Comparative modeling from the del(attP-Rel5)d9lacmutant proximal (top) and distal (bottom) limb bud cells. The red disk shows the position of region CS38-41 to be used as a reference point in all representations. The 3'-TAD and the 5'-TAD are indicated in $\mathbf{b}$ and $\mathbf{c}$. The TADkit tool was used to model Hi-C datasets from [34]. $\mathbf{f}$ Schemes of two inverted mutant lines where the HoxD cluster is disconnected from its adjacent regulatory domains: the inv(attP-Itga6)- and inv(Nsi-ltga6)mutant lines. On top, a Hi-C profile from limb cells with, below, the distribution of genes (gray) and regulatory regions (black) (chr2:7124000076320000). The positions of both the $5^{\prime}$ and $3^{\prime}-$ TADs are indicated with brackets. In the inv(Nsi-ltga6) allele, an inversion was generated between the Itga6 and the attP breakpoints [37] separating the HoxD cluster from the 3'-TAD. In the inv(Nsi-Itga6) allele, the inversion occurred between the Itga6 and the Nsi breakpoints [38], and hence, the HoxD cluster remains in contact with the 3'-TAD. In the latter inversion, a Hoxd1 1lac transgene (green flag) is inverted together with the DNA fragment

between the two regulatory domains were generally conserved between the two cell types, although with some minor differences.

We applied the same 3D modeling viewer to $\mathrm{Hi}-\mathrm{C}$ datasets obtained with limb bud cells from the HoxD $D^{\text {del(1-13)d9lac }}$-mutant mouse stock (hereafter $\operatorname{del}(1-13) d 9 l a c)$, which contains a deletion including the $\operatorname{HoxD}$ cluster [34] (see Additional file 1). In this mutant, the deleted DNA was substituted by a Hoxd9lac reporter transgene while the Evx2 and Lnpk genes remained present. In the absence of the HoxD cluster, the $5^{\prime}$-TAD and $3^{\prime}$-TAD were still observed as independent structures despite a substantial shortening of the distance separating them (Additional file 1B-C). A clear spatial contraction was nevertheless scored between the $5^{\prime}$ TAD and the first sub-TAD in the $3^{\prime}-\mathrm{TAD}$ until region CS38-41 (Additional file 1B, C, red disk).

We next used the HoxD del(attP-Rel5)d9lac (hereafter del(attP-Rel5)d9lac) Hi-C datasets from mutant limbs lacking ca. $350 \mathrm{~kb}$ of DNA including the HoxD cluster plus flanking regions (Fig. 1b, d, e). With this large deletion, the two TADs merged into one single structure (Fig. 1d, e) regardless of the cell type considered (distal or proximal limb bud), indicating that the TAD boundary had been entirely deleted. In this stock, the same Hoxd9lac transgene could be used as a transcriptional readout. The consolidation of both the 3 '-TAD and the 5 -TAD into one single structure was obvious up to the CS38-41 region, whereas the most telomeric located sub-TAD in the $3^{\prime}$-TAD was somewhat less engulfed (Fig. 1e). We also performed the $C$-score analysis and attributed the interacting domains according to the positive $C$-score values to obtain the distribution of compartments $\mathrm{A}$ and $\mathrm{B}$. We concluded that the position of the HoxD locus in compartment $\mathrm{A}$, as well as the general compartment distribution along chromosome 2, was virtually identical between distal and proximal cells, when both the wild-type and the del(attP-Rel5)d9lac datasets were considered (Additional file 1D). Given that the topological changes in $\operatorname{del}(\operatorname{attP}$-Rel5)d9lac were limited to those domains adjacent to the cluster, we decided to investigate the impact on the activity of these regulatory domains upon the aforementioned deletion or using inversions where the HoxD cluster was disconnected either from the $3^{\prime}$-TAD or from the $5^{\prime}$-TAD (Fig. 1f).

\section{Transcription at the HoxD locus in the absence of the HoxD cluster}

We assessed the transcription emanating from the lac $Z$ reporter transgenes by whole-mount in situ hybridization (WISH) on E11.5 fetuses, using a $\mathrm{LacZ}$ RNA probe and could identify both the distal and proximal limb domains in the two del(attP-Rel5)d9lac and $\operatorname{del}(1-13) d 9 l a c$ lines, though with subtle variations in their relative strengths (arrowheads Additional file 2A). Therefore, even in the complete absence of a TAD boundary (removed along with the deletion in the $\operatorname{del}($ attP-Rel5)d9lac allele), the functional partition of proximal and distal enhancers occurred in a close-tonormal manner, with a clear separation between the two expression domains. While the distal domain overlapped well with the wild-type HoxD distal limb pattern (see Hoxd10 and Hoxd11 wild-type WISH for a comparison), the proximal domain was somewhat different in shape and position from the wild-type $\operatorname{HoxD}$ pattern (Additional file 2A), resembling the expression pattern of the Hog lncRNA [34] thus likely indicating some enhancer reallocation due to the novel topology of the locus.

In order to have a more precise account of these local modifications in transcriptional responses following the fusion of the two TADs, we carried out RNA-seq for both proximal and distal cell populations in both control (Wt) and del(attP-Rel5)d9lac-mutant limbs at E12.5. In control proximal cells, transcripts were expectedly detected for Hoxd genes, for the flanking Lnpk and Mtx2 genes as well as for the Hog and Tog lncRNAs, two non-coding RNAs localized within the 3 '-TAD and normally responding to enhancers located in this domain [34, 39] (Fig. 2a, top). In control distal cells, 

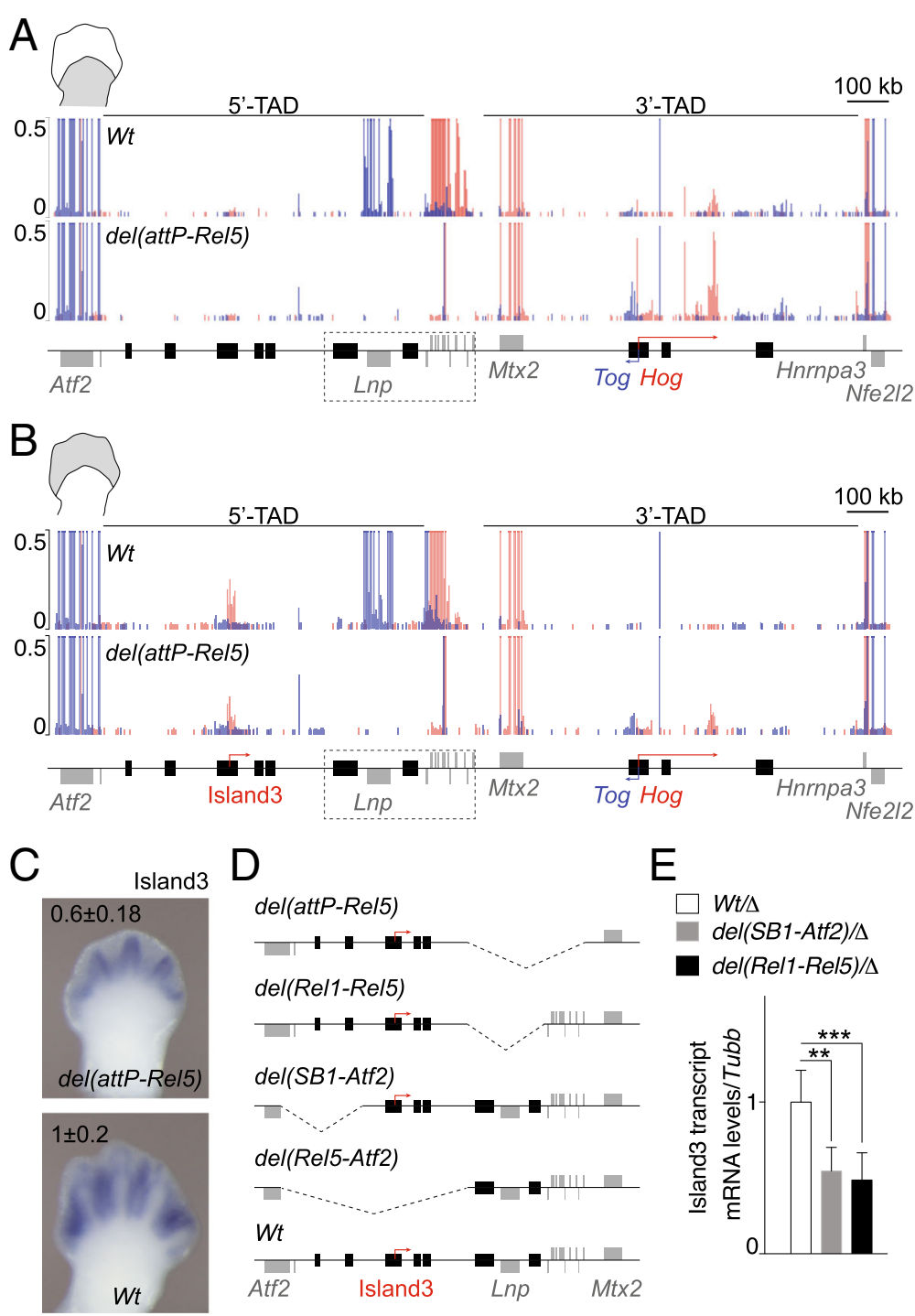

$\mathrm{E}$

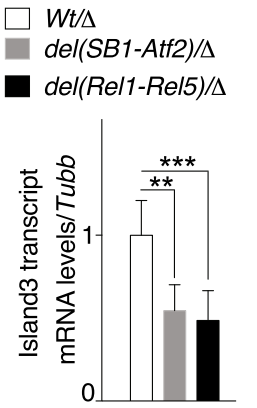

Fig. 2 Transcript profiles at the HoxD locus in both control (Wt) and del(attP-Rel5)d9lac-mutant limb buds. a, b Normalized RNA-seq profiles of control $(W t)$ and mutant proximal (a) and distal (b) limb cells. Values from forward (red) and reverse (blue) strands are merged into the same graph. The positions of various genes and of Island3 are shown below. The dashed rectangles depict the del(attP-Rel5)d9lac deletion. The isolated signal around Hoxd9 in the second tracks in $\mathbf{a}$ and $\mathbf{b}$ arises from the Hoxd9/lacZ reporter transgene present in the mutant line. The scale is set such that changes in non-coding regions can be better observed. $n=3$. c WISH of Island3 eRNA in both del(attP-Rel5)d9/ac and wild-type E12.5 forelimbs. qPCR values (mean \pm SD) are shown on the top of each image. $n=6$ for $W t$ and $n=4$ for del(attP-Rel5)d9lac. $\mathbf{d}$ Schemes of the various deleted regions of the mutant lines used in a to e. e qPCR of Island3 eRNAs in E12.5 distal limb cells in two distinct partial deletions of the 5'-TAD. The mutant lines used were del(SB1-Atf2) $(n=4)$ and del(Rel1-Rel5) $(n=9)$, both balanced by the del(Rel5-Atf2) allele (where Island3 is deleted, abbreviated by $\Delta$ in the legend). Results were compared to del(Rel5-Atf2)/ + samples as controls (white bar, $n=12$ ). Bars show mean \pm SD. Welch's $t$ test $*^{* *} p=0.0026$ and ${ }^{* * *} p<0.0001$

while the expression of the latter two lncRNAs was undetectable, digit-specific transcripts were scored over the Island3 region both by RNA-seq and by WISH (Fig. 2b, top; Additional file 2B), a region previously defined as a distal cell-specific enhancer [40]. Therefore, we used these non-coding RNAs ( $H o g$, Tog, and Island3) as proxies to evaluate the activity of their surrounding proximal versus distal enhancers in the two deletion alleles that removed all target Hoxd genes.
In proximal del(attP-Rel5)d9lac-mutant limb cells, the Hog and $\operatorname{Tog}$ RNA levels substantially increased (adjusted $p$ value from DESeq2 analysis of $1.75 \mathrm{e}-10$ and $6.72 \mathrm{e}-22$, respectively) while at the same time, the mRNA levels corresponding to the Mtx 2 and Atf2 housekeeping genes remained approximately the same (adjusted $p$ value $=1.00$ ) (Fig. 2a, bottom; Additional file 2C). Transcripts for Hoxd genes and Lnpk had expectedly disappeared after the deletion, yet a signal remained for Hoxd9 reflecting the transcription of the reporter gene left in place. Of note, the level of Island3 eRNA 
did not seem to increase in the deleted configuration. Therefore, while in the absence of target Hoxd genes, proximal enhancers within the former 3 '-TAD were partly re-allocated towards the neighboring $H o g$ and $T o g$ promoters, they did not seem to affect Island3 expression, despite the removal of the TAD boundary (Fig. 2a, bottom; Additional file 2C).

In distal del(attP-Rel5)d9lac-mutant limb cells, the level of Island3 eRNA decreased in the deleted configuration (Fig. 2b, c). While this transcript did not appear as differentially expressed in our RNA-seq whole genome analysis due to restrictive parameters $(46 \%$ reduction mutant versus control, adjusted $p$ value $=1.4 \mathrm{e}-4$; Additional file 2D), it showed a significant reduction by $\mathrm{qPCR}$ (40\% reduction mutant versus control, Welch's $t$ test $p$ value $=0.0166)$ and by WISH (Fig. 2c). To assess whether this reduction was due to the observed slight shrinking in the size of the presumptive digit domain in mutant limbs, we selected the ten genes with higher $\log 2$ fold change in RNA normalized read counts in distal versus proximal control limb cells. We next compared the normalized read counts of these mRNAs between mutant and wild-type distal limbs. Although some of them showed lower normalized read counts in del(attP-Rel5)d9lac, only $D b x 2$ and the 1810046K07Rik RNA appeared significantly reduced in the DESeq2 comparison (70\% decrease, adjusted $p$ value $=5.7 \mathrm{e}-28$, and $82 \%$ decrease, adjusted $p$ value $=5.3 \mathrm{e}-3$, respectively, Additional file $2 \mathrm{E}$ ). Therefore, it is likely that the decrease in Island3 transcripts was due to the deletion of both the GCR and Prox distal enhancers, as also suggested by the deletion of the Rel1 to Rel5 region (Fig. 2d, e).

A comparable outcome was observed as well in the deletion from SB1 to Atf2, which removes two different enhancers (islands 1 and 2) on the other end of the regulatory domain (Fig. 2d, e). Noteworthy, neither of the housekeeping transcription units was transcribed more efficiently. However, a significant increase in $H o g$ and Tog lncRNAs was scored, while these two genes are normally silent in distal cells where the 3 '-TAD has been switched off (Fig. 2b, Additional file 2D). Such an upregulation could illustrate either a weakening in the repression of the $3^{\prime}-\mathrm{TAD}$ in distal cells or novel interactions between distal enhancers located in former $5^{\prime}$-TAD and the two lncRNAs' loci, following the deletion of the TAD boundary.

Altogether, these results indicate that distal and proximal limb enhancers that are normally located in different TADs and which work in an antagonistic manner can achieve close-to-proper functional specificities when they are regrouped into a single fused TAD.

\section{Changes in chromatin marks after TADs fusion}

To confirm these observations, we looked at the acetylation of H3K27, a histone modification that positively correlates with transcriptional activity, and compared proximal and distal E12.5 limb bud cells derived from both control and $\operatorname{del}(a t t P-R e l 5) d 9 l a c$-mutant fetuses. In proximal cells, the distribution of H3K27ac marks in the mutant material was as in control (wild-type) cells (Fig. 3a). H3K27ac modifications were found enriched in the active $3^{\prime}$-TAD while depleted from the inactive 5'TAD. The amount of H3K27ac was slightly increased over a large region in the $3^{\prime}$-TAD in mutant cells, with a particular increase at the transcription start site of both Hog and Tog (Fig. 3a, 120\% increase, arrowhead) thus confirming the previously described increased in RNA levels (Fig. 2). The distribution of H3K27ac marks over the 5'-TAD was comparable in control and mutant proximal cells (Fig. 3a, see del versus $W t$ ).

In $\operatorname{del}($ attP-Rel5)d9lac-mutant distal cells, an increase in H3K27ac was scored at region CS38-41 (Fig. 3b, 75\% increase), which correlated with the activation of these two lncRNAs in these mutant cells, while they are normally silent in their wild-type counterparts (Fig. 2b). In addition, a strong increase in this histone $\mathrm{H} 3$ mark was scored in CS93 (Fig. 3b, arrow, 75\% increase), a region characterized as a proximal limb enhancer [15]. The general distribution of H3K27ac appeared slightly increased throughout the $3^{\prime}$-TAD in mutant cells when compared to control (Fig. 3b). This slight increase in the 3'-TAD activity was also noticeable when analyzing proximal mutant tissue. A striking effect was however observed in H3K27ac coverage over the 5' -TAD in mutant versus control distal cells. A substantial loss of H3K27ac was indeed scored over the regulatory regions island1, 2, 4, and 5 (Fig. 3b, about $40 \%$ decrease). This effect was not as evident over island3, i.e., in the region where the enhancer transcript was detected in both control and mutant distal cells (Fig. 2b). Therefore, in distal cells, the fusion of the two TADs and removal of target genes seemed to weaken the transcriptional activity in the $5^{\prime}$-TAD while maintaining the activity of the $3^{\prime}-\mathrm{TAD}$ well above the silencing observed in control distal cells.

To back up this observation, we looked at the distribution of H3K27me3 marks, which are associated with gene silencing. In control proximal limb cells, H3K27me3 were detected over the 3'-TAD at E12.5 (Fig. 3c), i.e., when this landscape is still functionally active, due to the presence of a large percentage of negative cells in the dissected material (see [33]). In distal cells, where the 3 ' -TAD is switched off, a robust increase was detected with a strong coverage of the entire domain (Fig. 3d). In proximal cells, H3K27me3 marks were also scored over the silent $5^{\prime}$-TAD regulatory islands, a labeling that mostly disappeared upon the activation of these regulatory islands in distal cells (Fig. 3c).

The H3K27me3 profiles obtained with the $\operatorname{del}($ attPRel5)d9lac-mutant limb buds were in agreement with the distributions of both the H3K27ac marks and the transcripts. In proximal mutant cells, the profile was globally similar to that seen in control cells showing no 


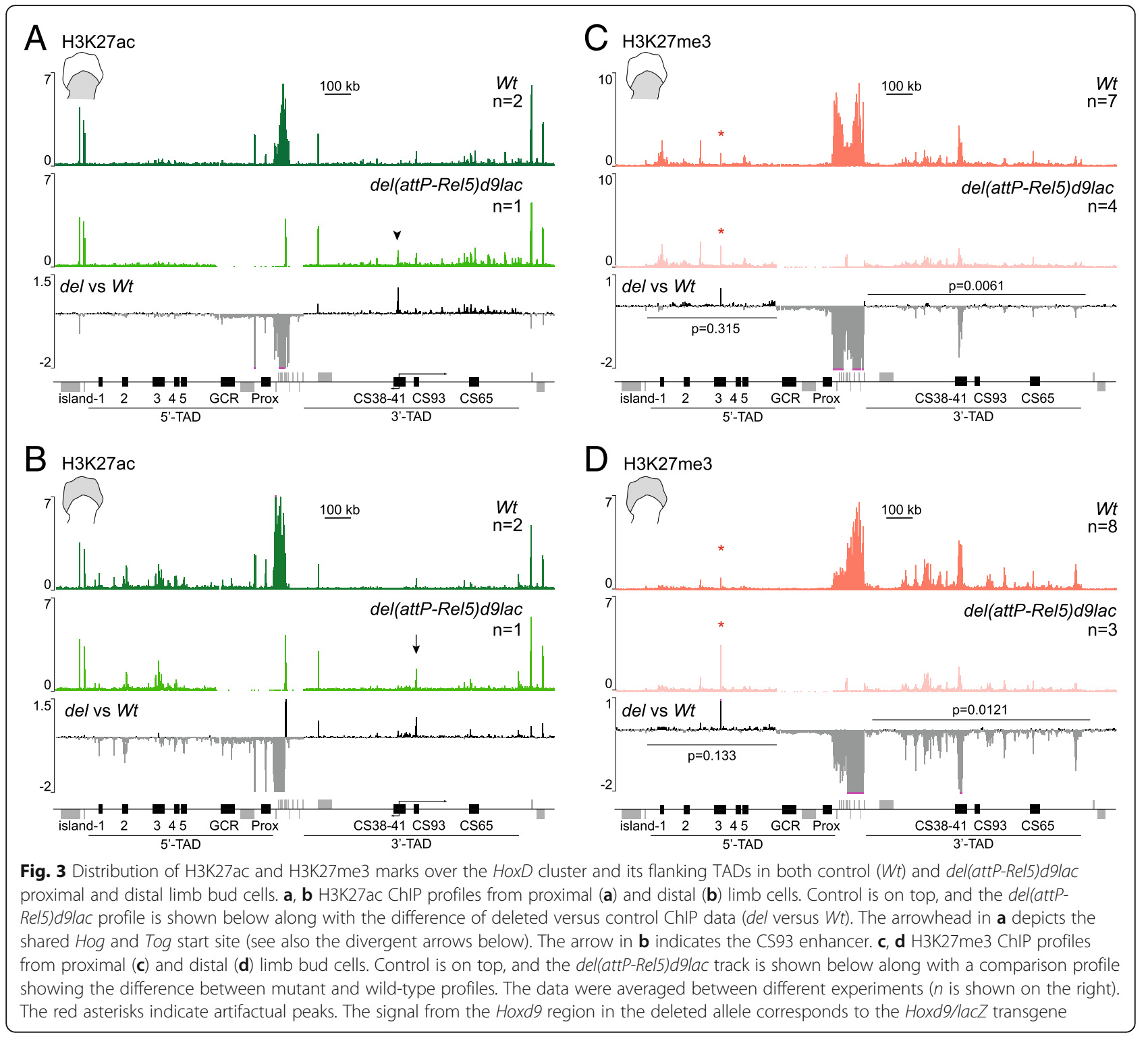

salient difference along the remaining part of the former $5^{\prime}$-TAD ( $6 \%$ increase, $p$ value $=0.32$ ), whereas a $22 \%$ decrease was scored over the remnant of the $3^{\prime}$-TAD $(p$ value $=0.0061)$. This decrease was mostly centered on region CS38-41, which showed a $51 \%$ decrease in the mutant (Fig. 3c). In distal cells, the same effect was scored, yet with a higher magnitude. H3K27me3 marks were indeed heavily depleted from the $3^{\prime}$-TAD $(50 \%$ reduction, $p$ value $=0.012$ ) whereas they were found mildly yet not significantly enriched all over the remaining $5^{\prime}$ TAD region containing the regulatory islands $(16 \%$ increase, $p$ value $=0.13$ ) (Fig. 3d). Therefore, these results confirmed that in mutant cells carrying the combined fused-TAD, the former 3 '-TAD landscape is globally overactive in distal cells at the expense of $5^{\prime}$-TAD enhancers, which appear less active than in their native context.

\section{Recruitment of PRC complexes at the HoxD cluster}

Polycomb repressive complexes (PRC1 and PRC2) are generally associated with lack of gene expression and usually recruited to the $\mathrm{CpG}$ islands close to the transcriptionally active regions [24, 41, 42]. In this context, the massive presence of H3K27me3 marks over the $3^{\prime}-\mathrm{TAD}$, a region largely devoid of coding units, raised the question of its recruitment. We looked at the presence of both EZH2 and RING1B, two components of PRC2 and PRC1, respectively. ChIP-seq experiments revealed that EZH2 was located mostly within the HoxD cluster (Fig. 4a). Outside the gene cluster, a weak enrichment was scored over region CS38-41 in proximal cells, which appeared even weaker in distal cells. Altogether, the two gene deserts were generally devoid of PRC2. A comparable 


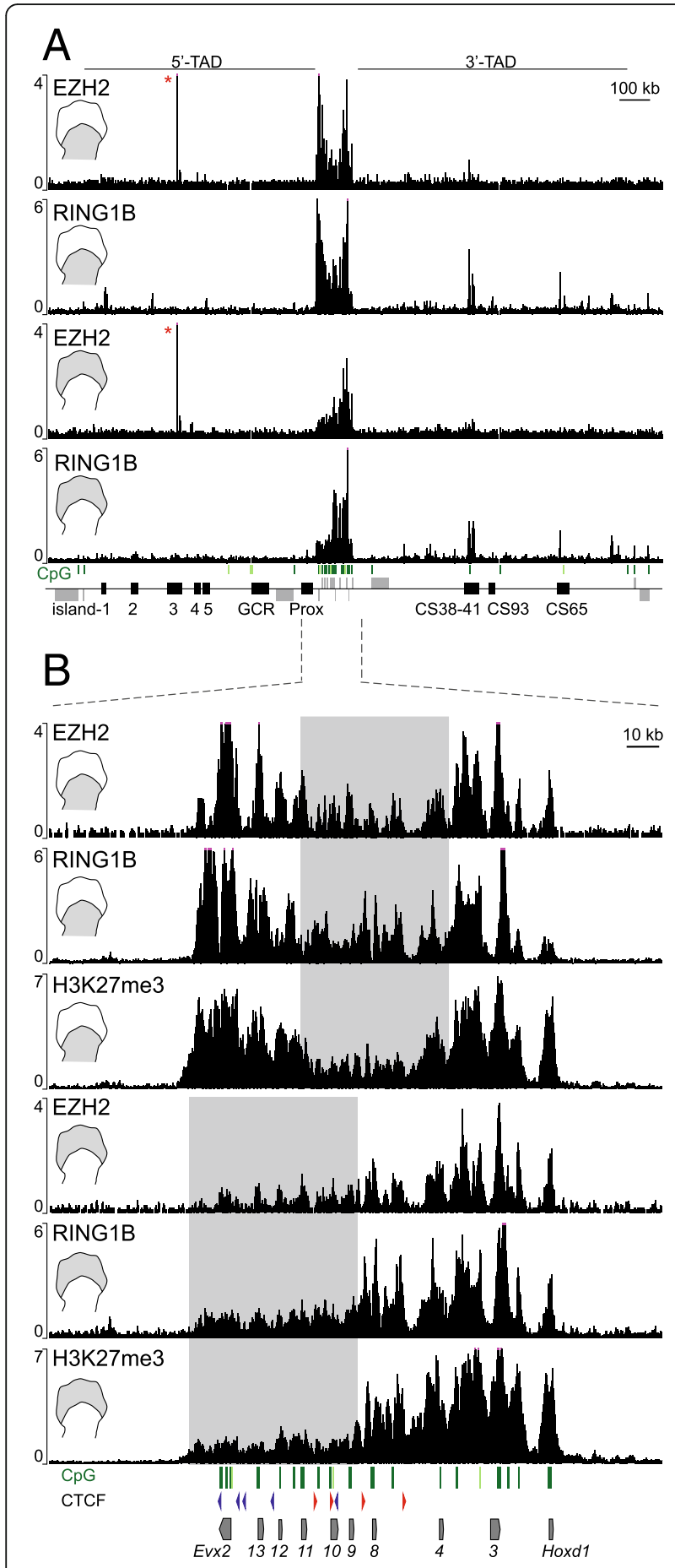

Fig. 4 Distribution of PRC1 (RING1B) and PRC2 (EZH2) over the HoxD cluster and regulatory landscapes in limb bud cells. a EZH2 and RING1B ChIP profiles in proximal (top two panels) and distal (bottom two panels) E12.5 limb bud cells. The CpG distribution is shown as green bars on top of the gene diagram. The red asterisk indicates an artifactual signal. b Magnification of the HoxD cluster showing the distribution of EZH2 and RING1B in proximal and distal limb cells. H3K27me3 ChIP tracks are shown for each tissue. The CpG islands are shown as green bars, and the CTCF and their orientations are depicted as blue (reverse strand) or red (forward strand) arrowheads conclusion was reached regarding the prevalence of signal at the cluster, with the analysis of the PRC1 component RING1B, even though some enrichment was detected on the gene deserts, generally over the 3'-TAD and particularly over the CS38-41 and CS65 regions, without any striking difference between distal and proximal cells (Fig. 4a). Some light differences were scored in the $5^{\prime}$-TAD, where a few regulatory regions appeared specifically decorated in proximal tissue but devoid of RING1B in distal tissue (compare Island1 and Island4 in Fig. 4a).

Within the HoxD cluster itself, the distribution of both EZH2 and RING1B nicely matched the coverage by H3K27me3 marks (Fig. 4b) [32, 33]. In proximal cells, the coverage was minimal over those genes active in response to the 3'-TAD enhancers (from Hoxd8 to Hoxd11, rectangle in Fig. 4b, tracks 1 and 2) while in distal cells, genes responding to $5^{\prime}$-TAD enhancers were bound only weakly by either PRC2 or PRC1 (from Hoxd13 to Hoxd9, Fig. 4b, rectangle in tracks 4 and 5). The EZH2 signals were significantly enriched at the CpG islands and over the coding regions, whereas the distribution of PRC1 was broader (Fig. 4b), suggesting a recruitment of PRC2 by CpG islands [24, 42, 43, 41].

Considering that H3K27me3 covered both Hox genes and their regulatory landscapes whereas PRC complexes were mostly recruited to the $\operatorname{HoxD}$ cluster itself, we wondered whether the reduction of H3K27me3 marks along the 3'-TAD in del(attP-Rel5)d9lac-mutant proximal cells could result from the mere absence of the HoxD gene cluster. To this aim, we used the engineered HoxD $D^{\text {inv(attP-Itga6) }}$ inversion (hereafter inv(attP-Itga6) where the HoxD cluster was disconnected from the 3'TAD and displaced circa $3 \mathrm{Mb}$ away while preserving both its integrity and its association with the $5^{\prime}$-TAD [37] (Fig. 1f).

We verified that the genomic interactions between Hoxd genes and the 3'-TAD were abrogated in this inv(attP-Itga6) inverted allele by performing a $4 \mathrm{C}$-seq analysis in mutant and control distal limb cells, with Hoxd4 and CS38 as viewpoints (Fig. 5a). Expectedly, the contacts established by Hoxd4 were no longer oriented towards the 3'-TAD in the inversion allele, when compared with control (Fig. 5a, tracks 1 and 2). In this inverted allele, interactions were now established de novo between Hoxd4 and a region around the Itga6 and Dlx1/Dlx2 genes, near the inversion breakpoint. Also, contacts with the $5^{\prime}$-TAD were slightly increased. Furthermore, when region CS38 was used as a viewpoint, interactions with the $\operatorname{HoxD}$ cluster were largely lost and most contacts remained within the 3 '-TAD itself (Fig. 5a, tracks 3 and 4).

In this inverted configuration, the global amount of H3K27me3 marks deposited over the 3'-TAD was 


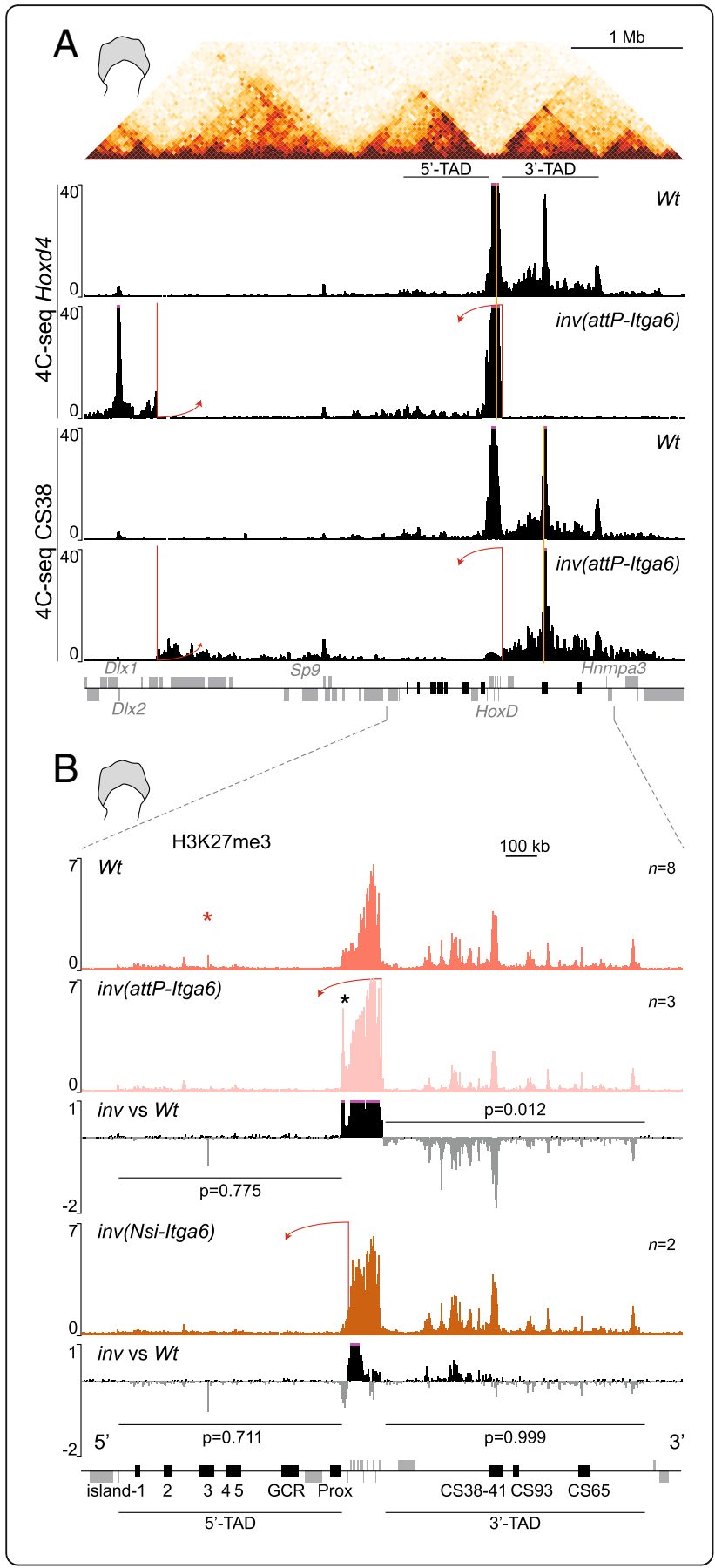

Fig. 5 Epigenetic changes after disconnecting the HoxD cluster from its flanking 3'-TAD. a On top, a Hi-C profile of distal limb bud cells shows the HoxD-associated TADs. The panels below show a comparison of 4C-seq tracks between control (Wt from [34]) and inv(attP-Itga6)-mutant distal limb cells. Either the Hoxd4 gene (top two panels) or the CS38 region (bottom two panels) was used as baits (yellow vertical bars). The red bars indicate the locations of the loxp sequences used to generate the inversion. After inversion, contacts between Hoxd4 and the 3'-TAD are all lost, while they barely change when region CS38 is used as bait. b H3K27me3 ChIP profiles in control (Wt) and either the inv(attPItga6) inversion (top two profiles) or the inv(Nsi-ltga6) inversion (bottom two profiles). Below each mutant track, a comparison between mutant and control data is shown. The red bars indicate the inversion breakpoints. In the inv(attP-Itga6) track, an additional peak appears at the $5^{\prime}$ extreme of the HoxD cluster (black asterisk), corresponding to an ectopic sequence introduced when building the attP breakpoint. The red asterisk indicates an artifactual signal. The number of replicates is shown for each track. Below each mutant track, a difference profile of mutant versus control signals is represented

substantially lower when compared to the control cells (Fig. 5b, tracks 1 and 2; Additional file 3). This decrease was not observed when another inversion was used as a control. In the HoxD $D^{i n v(N s i-I t g a 6)}$ allele (hereafter $i n v(N s i-$ Itga6) [38], the HoxD cluster remains in place yet the $5^{\prime}$ TAD is inverted towards the same Itga6 breakpoint (Fig. 1f). Therefore, these two inversions are identical except that one contains the $\operatorname{HoxD}$ cluster whereas the other does not (Fig. 5b, arrows in tracks 2 and 4; Additional file 3). In the inv(Nsi-Itga6) inversion allele, the enrichment of H3K27me3 over the 3'-TAD remained unchanged either in distal cells $(p$ value $=0.999)$, or in proximal cells ( $p$ value $=0.50)$, as was the case for the inv(attP-Itga6) allele (Fig. 5b and Additional file 3). Altogether, these results and those obtained with the $\operatorname{del}(a t t P-R e l 5) d 9 l a c$ allele suggest that the presence of Hoxd genes was necessary to achieve a full spread of H3K27me3 marks over the 3'-TAD, up to $800 \mathrm{~kb}$ in far-cis.

Of note, this effect was restricted to the 3 '-TAD, a conclusion reached after zooming out and looking at a $10-\mathrm{Mb}$ interval surrounding the $\operatorname{HoxD}$ cluster. In control distal cells, the distribution of H3K27me3 marks was enriched selectively over the 3'-TAD, terminating abruptly at its TAD boundary with no further telomeric spreading. In mutant $\operatorname{del}($ attPRel5)d9lac distal cells, despite the large reduction of H3K27me3 signals, the remaining coverage was also restricted up to the new telomeric boundary of the fusedTAD (Additional file 4A, B) without extending into neighboring TADs (R1 and R4 in Additional file 4A, 4B). Similar results were obtained when comparing the mutant inv(attP-Itga6). In all cases, though to a different extent, the TAD structure appeared to determine the extent of H3K27me3 spreading. 


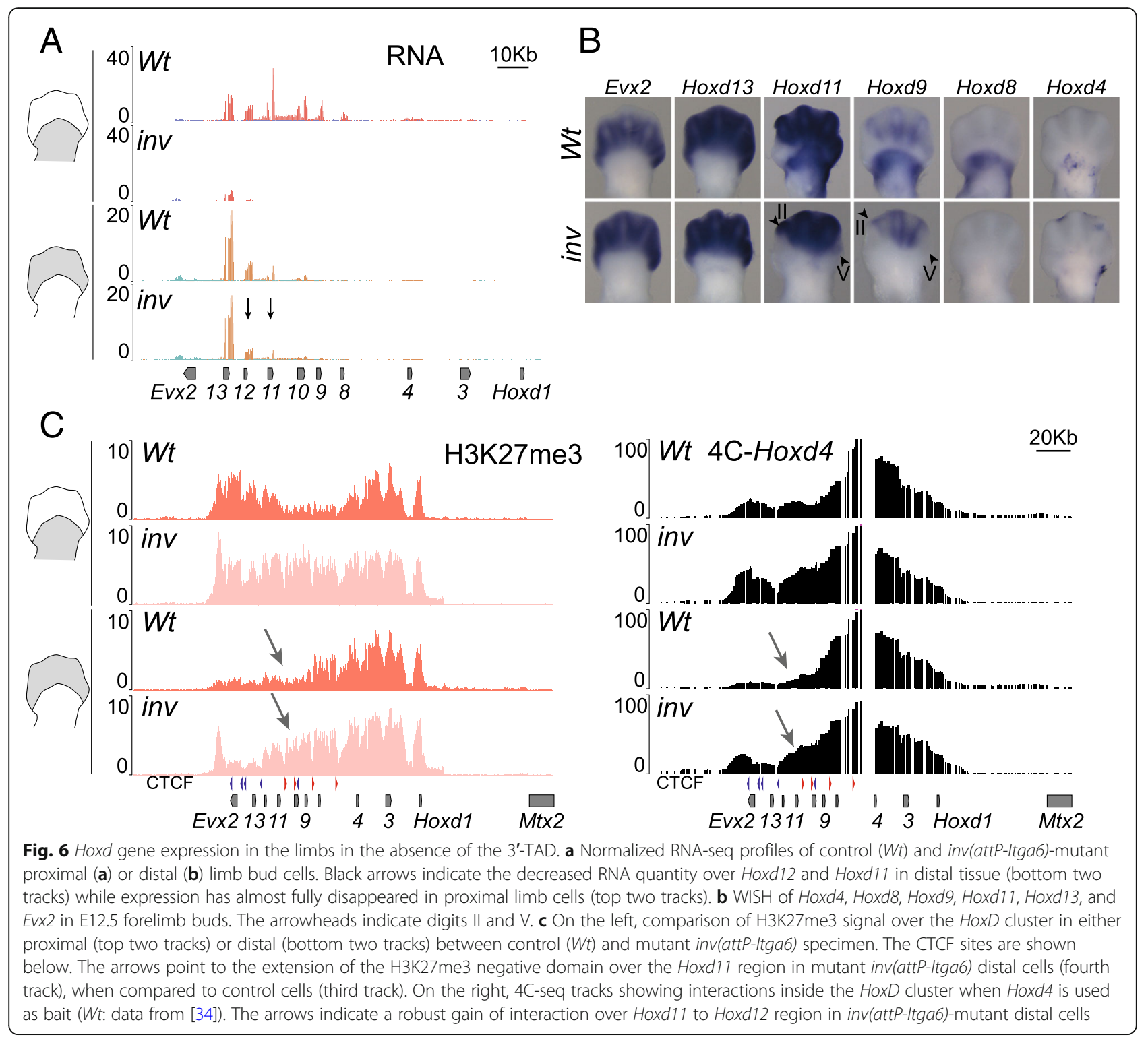

\section{H3K27me3 inheritance and clearance}

In the inv(attP-Itga6) allele, the 3'-TAD proximal enhancers were disconnected from their target Hoxd3 to Hoxd11 genes, similar to a previous case when a deletion of 3 '-TAD was used [33]. In both cases, the expression of these target genes was expectedly lost in proximal cells of the forelimb buds (Fig. 6a, b; see also [33]). Unexpectedly, however, both the quantity and distribution of Hoxd9 and Hoxd11 mRNAs were also reduced in distal cells (see digits II and V), where these genes are under the control of $5^{\prime}$-TAD enhancers (Fig. 6a, b, arrows and arrowheads, respectively). This surprising observation was explained by the lineage transmission, from proximal to distal cells, of H3K27me3 marks abnormally present in Hoxd genes in the absence of $3{ }^{\prime}$ TAD [33].
To further substantiate this possibility, we analyzed the precise distribution of H3K27me3 marks over the HoxD cluster in the $\operatorname{inv}($ attP-Itga6) allele. In proximal cells, we found a high and homogeneous coverage of this histone modification, from Hoxd1 up to Evx2, unlike in the control allele where the DNA interval between Hoxd8 and Hoxd11 was transcriptionally active and hence depleted from this mark (Fig. 6c, tracks 1 and 2). This homogeneous distribution of $\mathrm{H} 3 \mathrm{~K} 27 \mathrm{me} 3$ over the gene cluster in the mutant allele reflected the complete lack of Hoxd expression in proximal cells (Fig. 6a, tracks 1 and 2; Fig. 6b). In control distal cells, the region from Evx2 to Hoxd9 was depleted of H3K27me3 marks, as expected from the active regulation of the $5^{\prime}$-TAD enhancers.

In inverted mutant distal cells, however, an abnormally high H3K27me3 coverage was scored over the Hoxd9 to 
Hoxd11 region (Fig. 6c, arrow in track 4), which corresponded to the decrease in transcript levels observed for these genes in these cells (Fig. 6a, tracks 3 and 4). This increase in H3K27me3 was not observed in the inv( NsiItga6), where these genes are normally expressed in proximal tissue (Additional file 5). Because distal limb bud cells are the descendants in the lineage of proximal cells (see [44]), we explain this negative effect over the 5 '-TAD regulation by the transmission of H3K27me3 marks from proximal to distal cells. These marks were ectopically detected over the Hoxd4 to Hoxd11 region in proximal cells due to the lack of contacts between proximal enhancers and their target Hoxd genes thus preventing their transcriptional activation. Of note, Hoxd13 and Evx2 transcript levels remained unchanged in the mutant allele when compared to control.

We assessed whether this ectopic gain of H3K27me3 in proximal cells would translate into a change in the extent of the negative chromatin sub-domain formed at Hox loci by H3K27me3-enriched sequences [45, 46]. We carried out 4C-seq by using Hoxd4 as a viewpoint and noticed that in proximal cells, contacts established by Hoxd4 clearly extended over the $5^{\prime}$ part of the cluster in the mutant allele, in agreement with the gain of H3K27me3. These contacts were also observed, though to a slightly lesser extent, in mutant distal cells, again correlating with the persistence of H3K27me3 marks (Fig. 6c, arrow in track 4).

\section{Discussion}

During limb development, the two TADs associated with the $\operatorname{HoxD}$ cluster are either transcriptionally active or repressed, in an exclusive manner. Initially, the 3'-TAD enhancers are active and control the first wave of Hoxd transcription in early limb buds. Subsequently, these enhancers activate Hoxd genes in proximal structures such as the forearms [33]. In the second phase, the $5^{\prime}$-TAD enhancers become activated in distal limb cells (the future hands and feet) while the 3'-TAD concomitantly terminates operating and becomes covered by negative H3K27me3 marks [33, 40]. This bimodal regulation in TAD activities is necessary to organize each of the proximal and distal Hox expression domains, which are essential for proper limb development [47-50].

\section{A fused-TAD}

Previous studies of this functional switch between these two TADs have suggested that they could represent coherent and independent regulatory units, i.e., that the $3 \mathrm{D}$ structure itself may participate in the global functional output of the system. In this view, a TAD could be either functionally permissive or refractory to the implementation of all the enhancers it may contain [32] thus representing an additional regulatory layer. In the case of both $3^{\prime}$-TAD and $5^{\prime}$-TAD, only one of them is licensed to work at a time since the presence of HOX13 proteins, partly determined through the activation of the $5^{\prime}$-TAD, leads to the repression of the $3^{\prime}$-TAD [32]. We wondered how this functional exclusivity would translate after the fusion of the two structures, in a situation where both proximal and distal enhancers would be included in the same fused-TAD. In this fused-TAD indeed, several enhancers normally present in the $5^{\prime}$-TAD, i.e., with a distal specificity, are now located along with the enhancers normally displaying a proximal specificity due to their location within the $3^{\prime}$-TAD. Since their target Hoxd genes were absent, we assessed their functionality by using three transcription units as readouts: an eRNA encoded by Island3 within former $5^{\prime}$-TAD, the $H o g$ and $T o g$ lncRNAs encoded within former 3'-TAD and a Hoxd $9 /$ lac $Z$ reporter transgene positioned exactly between the former two TADs.

The analysis of lacZ mRNA revealed the presence of distinct proximal and distal expression domains, suggesting that the presence of the two kinds of enhancers in the same fused-TAD did not drastically affect neither their global functional specificities nor their mode and sequence of action. However, the proximal domain was distinct from what is normally observed in the wild-type limbs, despite the remaining presence of all known proximal enhancers in the two deleted alleles. In fact, it resembled in its position and shape to the expression domain of the lncRNA $H o g$, which is located within the $3^{\prime}$-TAD surrounded by proximal enhancers. In this case, the absence of target genes and their associated CTCF sites may have led to reallocations in enhancer-promoter contacts, as also suggested by the upregulation of $\mathrm{Hog}$ and $T o g$ lncRNAs in proximal mutant cells. Therefore, the final transcription readout of 3 '-TAD enhancers may slightly vary in space and time depending on how the target promoters are organized and on their local topology.

In addition, $H o g$ and $T o g$ transcripts were scored in mutant distal cells, while completely switched off in control distal cells. We interpret this as a response of these lncRNAs to the remaining $5^{\prime}$-TAD enhancers, in the absence of the TAD boundary. Also, the global repression of the 3'-TAD in mutant distal cells was not implemented as efficiently as in control cells, thus contributing to this light upregulation. At this point, it is difficult to associate the upregulation of these lncRNAs either with the lack of $3^{\prime}$-TAD repression or to de novo established promoter-enhancer interactions, given that both events would have a similar transcription outcome. However, when comparing to other remaining genes, these effects were specific for $H o g$ and Tog, and hence, it seems that they may engage with active enhancers. Housekeeping genes located within or in the vicinity of 
the former 3'-TAD, such as Mtx2, Hnrnap3, or Atf2, were transcriptionally unaffected after fusion of the TADs, as these genes generally escape the regulations exerted by global enhancers in these landscapes.

In parallel with the maintenance of proximal enhancer activity in distal cells of the fused-TAD, the level of Island3 eRNAs was slightly reduced. While this RNA was exclusively present in distal cells, the same regulatory region showed a diminution of its transcriptional activity after merging of the two TADs, as if the fused-TAD was globally pushed towards a proximal type of regulation. A clear distal domain was nevertheless still detected with the lac $Z$ expression pattern, demonstrating the activity of at least some distal limb enhancers and suggesting that the reduction in Island3 eRNA level may also be caused by the deletion of some distal enhancers in the fused-TAD.

This tendency of the fused-TAD to adopt a type of regulation globally more proximal than distal was reinforced by the analyses of chromatin marks. In distal cells, the fusion between the two TADs was indeed accompanied by a decrease in H3K27ac coverage in several enhancers located in the former $5^{\prime}$-TAD. In contrast, H3K27ac marks in mutant distal cells were more abundant in the former 3'-TAD region, i.e., over proximal enhancers, than in control distal cells where these marks rapidly disappear [33]. In general terms, however, H3K27ac deposition associated with enhancer activation in mutant cells was still observed as in control cells, indicating that the former 3 '-TAD enhancers were still active in proximal limb bud cells and the former $5^{\prime}$-TAD enhancers in distal cells. The difference was observed in the balance between these two types of regulations rather than in their implementation.

The profile of H3K27me3 marks confirmed these observations. In distal cells where the 3'-TAD is normally inactive, the amount of H3K27me3 was significantly reduced in mutant versus control cells, as if the "proximal regulation" had not been entirely switched off in distal cells. In parallel with both the decrease of Island3 eRNAs level and the decrease in H3K27ac, the distribution of H3K27me3 marks appeared increased in the former 5'-TAD. Altogether, these results suggest that when mixed into a single fused-TAD, the proximal regulation tends to take the lead over the distal regulation, with proximal enhancers being active for too long, even in distal cells where distal limb enhancers seem to be somewhat under-active. A potential mechanism may involve the reported effect of HOX13 proteins in the termination of 3 '-TAD regulation, combined with the novel chromatin architecture of the fused-TAD. In the absence of HOXD13 proteins, deleted from the fusedTAD, the dose of HOXA13 should be sufficient to secure the repression of $3^{\prime}$-TAD and thus to implement the switch in regulations [32]. However, the new chromatin configuration of this part of 3 '-TAD when included in the fused-TAD may affect the negative function of HOXA13, leading to a partial inhibition only and hence to an improper switch off of proximal enhancers.

\section{TAD-specific and long-range effect of PRC silencing}

Our results also provide some indications as to how PRC silencing propagates in-cis at a distance (see [51, 52]). Within the HoxD cluster itself, we show that PRC2 recruitment selectively occurs at the $\mathrm{CpG}$ islands, as previously proposed (e.g., [24, 52]). In addition, however, H3K27me3 marks were found throughout the 3 '-TAD (over ca. $800 \mathrm{~kb}$ ) in distal cells, where proximal enhancers have terminated their function, even though H3K27me3 marks were shown not to spread outside the HoxD cluster in a linear manner [53]. In the $\operatorname{del}($ attPRel5)d9lac deletion, in the almost complete absence of CpG islands in and around the HoxD cluster, the enrichment by H3K27me3 marks in the 3'-TAD was severely reduced in distal cells, indicating that indeed the recruitment of PRC2 complexes over the HoxD cluster was mandatory to start covering the telomeric regulatory landscape by H3K27me3 marks, concomitantly to its functional inactivation. Some H3K27me3 coverage was nevertheless detected in the $5^{\prime}$-TAD and more substantially in the $3{ }^{\prime}-\mathrm{TAD}$, perhaps due to the presence of both the Hoxd9/lacZ reporter transgene and the Hog and Tog transcription start sites.

The coverage by H3K27me3 marks in control distal cells outside the $\operatorname{HoxD}$ cluster itself, i.e., in a region that is not particularly enriched in PRC2, exactly matched the extent of the 3'-TAD containing those Hoxd genes inactivated in distal cells and hence heavily covered by PRC2, PRC1, and H3K27me3. Such an effect was not scored in any other region in the $10 \mathrm{Mb}$ surrounding the HoxD locus. This result suggests that the global inactivation of the $3^{\prime}$-TAD regulation in distal cells [32] is accompanied by a TAD-specific coverage of H3K27me3 marks, up to the telomeric TAD boundary where the presence of these negative marks abruptly stops (see also $[54,55])$. Therefore, the TAD structure itself may dictate the extent of coverage by H3K27me3 marks, after the recruitment of PRC2 on those Hoxd genes switched off in these distal cells and included into this TAD.

\section{Heritability of polycomb-associated gene silencing}

During the replication of H3K27me3-labeled DNA sequences, daughter cells inherit this histone modification from their parental cell $[51,56,57]$. Since limb development occurs mainly through a distal outgrowth, the distal cells where $5^{\prime}$-TAD enhancers are at work derive from proximal cells that were previously under the 
control of $3^{\prime}$-TAD enhancers. In the latter cells, the central part of the HoxD cluster is active and hence Hoxd9, Hoxd10, and Hoxd11 are devoid of H3K27me3 marks, whereas Hoxd12 and Hoxd13, which are located on the other side of the TAD boundary are silent and thus covered by H3K27me3 marks [33].

When these cells start to implement the $5^{\prime}$-TAD distal regulation, H3K27me3 marks are erased from both Hoxd13 and Hoxd12, the major targets of 5'-TAD enhancers, which are transcribed at high levels. Hoxd11 and Hoxd9, which are devoid of H3K27me3 marks also become transcribed in distal cells, even though their genuine function in these cells has not been unequivocally demonstrated [39]. In the absence of 3 '-TAD regulation in inv(attP-Itga6) proximal mutant cells, the entire HoxD cluster is heavily covered by $\mathrm{H} 3 \mathrm{~K} 27 \mathrm{me} 3$ marks since all Hoxd genes are silenced. When these mutant distal cells start to implement the $5^{\prime}$-TAD regulation, the H3K27me3 marks covering Hoxd13 and Hoxd12 are removed with the same kinetics as in wildtype distal cells, due to a comparable transcriptional context. However, Hoxd11 and Hoxd10 transcription onset is severely delayed when compared to control distal cells, as these genes were inherited in a silenced rather than active state, covered by H3K27me3 marks [33]. In this latter case, the strength of distal limb enhancers and the proximity of Hoxd13 and Hoxd12 likely lead to a progressive removal of PRC silencing and a weak and delayed activation of both Hoxd11 and Hoxd10 in distal cells. This observation illustrates both the capacity for cells to memorize their coverage in H3K27me3 marks in a physiological context and the labile aspect of polycomb silencing, which can be efficiently removed through a strong transcriptional activation.

\section{Conclusions}

From this study, we conclude that proximal and distal limb enhancers, which are normally segregated between the two TADs flanking the $\operatorname{HoxD}$ cluster, were not dramatically affected neither in their activation nor in their specificities, when their target genes were deleted and the two TADs merged into a single chromatin interaction domain. However, in the fused TAD, the proximal regulation seems to take the lead over the distal regulation. Secondly, these results suggest a mechanism whereby the silencing of remote enhancers is accompanied by a far-cis spreading of polycomb group proteins after being recruited for the most part at the HoxD cluster itself. Finally, we conclude that active genes are more readily accessible to a subsequent enhancer regulation when compared to silenced genes, illustrating the potential importance of polycomb-associated chromatin marks in the proper timing of gene activation during development.

\section{Methods}

\section{Animal experimentation and mouse strains}

Genetically modified mice were kept on a (Bl6XCBA) background and crossed in heterozygosis. Distal and proximal forelimb tissues were dissected and processed from E12.5 mouse embryos. All mutant mice used in this study and their genotyping strategies have been previously described in [34, 37, 40]. Homozygous mutant embryos were obtained by crossing heterozygous mice.

\section{D modeling of Hi-C datasets}

$\mathrm{Hi}-\mathrm{C}$ original datasets from wild-type $H o x D^{\text {del(1-13)d9lac }}$ and HoxD $D^{\text {del(attP-Rel5)d9lac }}$ were obtained from [34] (GEO accession: GSE101715). Three-dimensional modeling of the normalized $40 \mathrm{~kb}$ binned $\mathrm{Hi}-\mathrm{C}$ matrices was performed by means of the model_and_analyze.py script from the TADbit v0.2.0.58 software [36] in chr2: 73800001-75760000 (wild-type coordinates $\mathrm{mm} 10$ ). We generated 500 models for optimization and 5000 for modeling, and matrix columns showing no interactions were not filtered out. We visualized the model with TADkit using the Virtual Research Environment (https://vre.multiscalegenomics.eu/home/) [58]. Region CS38-41 (wild-type coordinates in mm10, chr2: 75120051-75165771) was used as a reference mark in the $3 \mathrm{D}$ reconstructed $\mathrm{Hi}-\mathrm{C}$ model. The analysis of $\mathrm{A} / \mathrm{B}$ compartments was performed using CscoreTool [59]. Briefly, the cis valid pairs on chr2 [34] of the wild-type and mutant $\mathrm{Hi}-\mathrm{C}$ mapped on mm10 were converted to the same format as the $\mathrm{Hi}-\mathrm{C}$ summary file format for HOMER. Then, CscoreTool was executed using $40 \mathrm{~kb}$ bins and a minDis of $1 \mathrm{Mb}$. The values of the bedgraphs with $C$-scores were inverted when they began with positive values. All bedgraphs were then loaded in the UCSC browser.

\section{RNA extraction, RNA-seq, and qPCR}

Limb tissue was dissected, placed in RNAlater (Invitrogen), and directly frozen at $-80^{\circ} \mathrm{C}$ until further processing. After genotyping, RNA was extracted from individual samples using RNAeasy Micro Kit (QIAGEN). Libraries were prepared from $100 \mathrm{ng}$ of total RNA using the TruSeq Stranded mRNA protocol and sequenced on a HiSeq 2500 machine (100 bp reads, single end). Sequencing data were treated using the facilities of the Scientific IT and Application Support Center of EPFL. The gtf file used for STAR and cufflinks, based on Ensembl version 94 annotations, is available on figshare [60]. Adapters were removed using cutadapt (v1.16) and aligned onto mm10 using STAR [61] with ENCODE parameters. Normalized read counts were obtained by DESeq2 analysis, which was performed with default parameters. Genes with absolute $\log 2$ fold change above 1.5 and adjusted $p$ value below 0.05 were considered as 
significant ([62] version 1.22.1). For Hox $D^{\text {del(attP-Rel5)d9lac }}$ and their associated control samples, three biological replicates were used for each genotype and tissue. For plotting distal RNA-seq comparisons between wild type

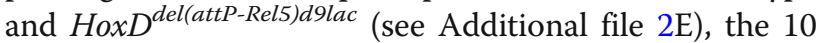
most differentially expressed genes in distal wild-type tissue were ranked and subsequently compared between mutant and control samples. For HoxD $D^{\text {inv(attP-Itga6) }}$ RNAseq, only one sample was used per tissue and genotype. Track profiles show the mean of the coverage of uniquely mapped reads normalized to the number of uniquely mapped reads. They were obtained with the UCSC browser. For qPCR, purified RNA was retrotranscribed with Promega GoScript Reverse Transcriptase (Promega). Custom SYBR probes were used for quantitative real-time PCR (qPCR) in a QuantStudio 5384-well block machine. Island3 primers were forward: TTCC ATCACAGGAGAGTCGTTG and reverse: AGGT GGGAACATGGACTGAAAG. All other primers were described in $[39,63]$. qPCR fold inductions were considered significant when Welch's $t$ test statistical analyses showed $p$ value $<0.05$.

\section{C-seq experiments}

The limb samples used in this study were dissected from E12.5 forelimb buds for all wild-type and mutant lines. The samples were processed as in [34]. Briefly, cellular suspensions were filtered and fixed using a $2 \%$ formaldehyde/10\% FBS/PBS solution for $10 \mathrm{~min}$. NlaIII (NEB) was used as the first cutter and DpnII (NEB) as the second cutter. DNA libraries were prepared using 12 to 14 independent PCR reactions with $100 \mathrm{ng}$ of DNA on each. Sequencing was performed by multiplexing several independently barcoded viewpoints. 4C-seq data were analyzed using the HTSstation web interface [64]. They were normalized to the distribution of reads on a $10-\mathrm{Mb}$ window, and the profiles were smoothened using a window of 11 fragments. 4C-seq data from wild-type tissue was taken from GEO (GSE101717). Data for the CS38 viewpoint were taken from GSM2713679 and for the Hoxd4 viewpoint from GSM2713671 and GSM2713672.

\section{Chromatin immunoprecipitation (ChIP)}

For all samples, limb tissues were dissected and directly fixed with $1 \%$ formaldehyde in PBS for $10 \mathrm{~min}$ at room temperature, followed by 3 min incubation with Stop Solution from the ChIP-IT High Sensitivity Kit (Active Motif). The samples were then washed 3 times with working Washing Solution (ChIP-IT, Active Motif) and then snap-frozen in liquid nitrogen and stored at $-80^{\circ} \mathrm{C}$ until further processing. After genotyping, the samples were pooled according to the required cell number. The total amount of tissue used for each line was different due to the size variations of the limb buds. Limb tissues were disrupted with a polytron device, lysed in RIPA buffer or Prep Buffer (ChIP-IT, Active Motif), and sonicated in Diagenode Bioruptor Pico. All H3K27ac ChIP experiments were processed as ChIP-seqs using the reagents from ChIP-IT High Sensitivity Kit (Active Motif). IPs were performed in parallel technical duplicates with 11 to $14 \mu \mathrm{g}$ of chromatin on each. Antibody incubation was performed overnight on a final volume of $1.5-2 \mathrm{ml}$ dilution buffer $(0.1 \%$ SDS, $50 \mathrm{mM}$ Tris- $\mathrm{HCl} \mathrm{pH} \mathrm{8,} 10$ $\mathrm{mM}$ EDTA $\mathrm{pH}$ 8, and proteinase inhibitors), including $2 \mu \mathrm{l}$ of H3K27ac antibody (Diagenode C15410196) at $4{ }^{\circ} \mathrm{C}$ on a rotating platform. Agarose beads were added for 3 to $4 \mathrm{~h}$ at $4{ }^{\circ} \mathrm{C}$. Washes were performed on column, and DNA purification was carried out by phenolchloroform extraction. The technical replicates were merged and yielded 1.5 to $2 \mathrm{ng}$ of chromatin, which were used to generate DNA libraries using the TruSeq ChIP library preparation kit. The number of biological replicates used for H3K27ac ChIP experiments is shown in the figure. RING1B ChIP experiments were processed as for ChIP-seq using $4 \mu \mathrm{l}$ of RING1B antibody (Active Motif 39664) and following the protocol described in [32]. Three and two biological replicates were processed for distal and proximal RING1B ChIP experiments, respectively.

All H3K27me3 and EZH2 ChIP were performed following the ChIPmentation protocol [65]. Around 0.1 to 0.4 million cells were used for each IP on a final volume of 800 to $1000 \mu$ l of RIPA-LS buffer $(10 \mathrm{mM}$ Tris- $\mathrm{HCl}$ pH 8, $140 \mathrm{mM} \mathrm{NaCl}, 1 \mathrm{mM}$ EDTA pH 8, 0.1\% SDS, 0.1\% sodium deoxycholate, $1 \%$ Triton X-100, and proteinase inhibitors), to which $2 \mu \mathrm{l}$ of H3K27me3 (Millipore 17622) or EZH2 (Diagenode C15410039) antibodies were added. The samples were incubated for at least $2 \mathrm{~h}$ with Dynabeads Protein A (Invitrogen 10001D) rotating at $4{ }^{\circ} \mathrm{C}$. Washes were performed as follows: two times RIPA-LS, two times RIPA-HS $(10 \mathrm{mM}$ Tris- $\mathrm{HCl} \mathrm{pH} 8$, $500 \mathrm{mM} \mathrm{NaCl}, 1 \mathrm{mM}$ EDTA pH 8, 0.1\% SDS, $0.1 \%$ sodium deoxycholate, $1 \%$ Triton $\mathrm{X}-100$, and proteinase inhibitors), two times RIPA- $\mathrm{LiCl}(10 \mathrm{mM}$ Tris- $\mathrm{HCl} \mathrm{pH} 8$, $250 \mathrm{mM} \mathrm{LiCl}, 1 \mathrm{mM}$ EDTA pH $8,0.5 \% \mathrm{NP}-40,0.5 \%$ sodium deoxycholate, and proteinase inhibitors), and once with $10 \mathrm{mM}$ Tris- $\mathrm{HCl} \mathrm{pH}$. The beads were resuspended in $24 \mu$ of tagmentation buffer $(10 \mathrm{mM}$ Tris $\mathrm{pH}$ $8,5 \mathrm{mM} \mathrm{MgCl} 2,10 \%$ dimethylformamide) and $1 \mu \mathrm{l}$ of Tn5 transposase (Illumina 15027865, from Nextera DNA Library Prep Kit 15028212) and transferred to PCR tubes, which were then incubated at $37^{\circ} \mathrm{C}$ for $5 \mathrm{~min}$ in a thermocycler. The samples were then resuspended and washed twice in $1 \mathrm{ml}$ of RIPA-LS and twice in $1 \mathrm{ml}$ TE buffer ( $10 \mathrm{mM}$ Tris-Hcl pH $8,1 \mathrm{mM}$ EDTA $\mathrm{pH}$ 8). The beads were magnetized, and DNA was eluted in ChIP elution buffer $(10 \mathrm{mM}$ Tris- $\mathrm{HCl} \mathrm{pH} 8,5 \mathrm{mM}$ EDTA pH $8,300 \mathrm{mM} \mathrm{NaCl}, 0.4 \%$ SDS) with $2 \mu \mathrm{l}$ of 
proteinase $\mathrm{K}(20 \mathrm{mg} / \mathrm{ml}$ stock) and then incubated for 1 $\mathrm{h}$ at $55^{\circ} \mathrm{C}$ and $6 \mathrm{~h}$ to overnight at $65^{\circ} \mathrm{C}$. After decrosslinking, the supernatant was recovered and the beads were resuspended again in $19 \mu \mathrm{l}$ ChIP elution buffer with $1 \mu \mathrm{l}$ of proteinase $\mathrm{K}$ and left $1 \mathrm{~h}$ at $55^{\circ} \mathrm{C}$. The two supernatants were combined and purified with MinElute kit (Qiagen) in $22 \mu \mathrm{l}$ of EB buffer. Relative quantitation was performed using SYBR Green (as in [65]) using $2 \mu \mathrm{l}$ of DNA. Libraries were amplified according to the $\mathrm{Cq}$ values obtained in the previous step (12 to 14 cycles for both sets of samples), purified using Agentcourt AMPureXP beads (Beckman Coulter A63880) and eluted in $15 \mu \mathrm{l}$ of water. DNA sequencing was performed in HiSeq 2500 or HiSeq 4000 machine as $50 \mathrm{bp}$ single reads or $100 \mathrm{bp}$ single reads. Only one experiment per tissue was performed for the EZH2 ChIP. The number of biological replicates used for H3K27me3 ChIP experiments is shown in the figure.

\section{ChIP analysis}

Analyses were performed using the facilities of the Scientific IT and Application Support Center of EPFL. Sequences were filtered, and adapters were removed using cutadapt (v1.16) [66] with parameters -m 15 -q 30 -a CTGTCTCTTATACACATCTCCGAGCCCACGAGAC for ChIPmentation and -a GATCGGAAGAGCACACGTCTGAACTCCAGTCAC for ChIP-seq. Reads were mapped on mm10 using bowtie2 (v2.3.4.1) using default parameters [67]. Only reads with a mapping quality above 30 were kept. A profile was obtained with macs2 [68] (version 2.1.1.20160309 option -extsize 300). Bedgraphs were normalized to their number of million tags used in the profile, and replicates were merged using the tool unionbedg (bedtools v2.27) [69]. The profiles were loaded in the UCSC browser with "mean" as windowing function. The difference profiles were calculated using unionbedg. In order to quantify the gain or loss of chromatin marks in the 3'-TAD, and in the CS38-41 region, the number of reads falling into their respective intervals (chr2:74781516-75605516 for the 3'TAD and chr2:75120051-75165771 for the CS38-41 region) were assessed after duplicate removal by picard (http://broadinstitute.github.io/picard/ version 2.18.14) [70] using the multiBamSummary function from deeptools [71]. For the 5'-TAD, two different ranges were taken into account depending on whether the full regulatory domain was intact in the mutant configuration: chr2:73914154-74636454 when comparing wild type, $H o x D^{\text {inv(attP-Itga6) }}$, and HoxD $D^{\text {inv(Nsi-Itga6) }}$ alone (Fig. 5, Additional file 3) and chr2:73914154-74422050 whenever HoxD del(attP-Rel5)d9lac was included in the comparisons (Fig. 3, Additional file 4). Also, for the 5'-TAD, the reads falling into the region of the artifactual peak, which is due to a PCR contamination (chr2:74207282-
74208158), were excluded in all datasets. The counts were normalized to the number of reads in the input bam file, and the significance (Wilcoxon rank-sum test) was assessed by the function wilcox.test in R (https:// www.R-project.org).

\section{Whole-mount in situ hybridization and beta-galactosidase staining}

Island3, Hoxd4, Hoxd8, Hoxd9, Hoxd11, Hoxd13, and Evx2 WISH were performed following the protocol described in [72]. The DNA fragment for Island3 probe was amplified from purified genomic DNA using primers GCAGGAATGACAGACAGGCA (Fw) and ACAGAGGTGGGAACATGGAC (Rv) and cloned into pGEM-T easy vector (Promega A1360). Betagalactosidase staining was performed as in [34]. Hoxd4, Hoxd8, Hoxd9, Hoxd11, Hoxd13, and Evx2 probes were as in [73].

\section{Additional files}

Additional file 1: 3D-representation of the HoxD locus in del(1-13)d9lacmutant limb buds. (A) Hi-C map showing the presence of both TADs on either side of the HoxD locus in del(1-13)d9lac proximal limb cells and its associated genes (gray boxes) and regulatory regions (black and red boxes) ( $B, C$ TADkit-derived 3D representation of $\mathrm{Hi}-\mathrm{C}$ datasets [34] obtained for distal (B) and proximal (C) limb cells processed from del(113)d9lac-mutant mice. The CS38-41 region is shown as a red disk in the $3 \mathrm{D}$ models to be used as a reference point. In this deletion allele, both TADs are still visible unlike in the larger del(attP-Rel5)d9lac deletion shown in Fig. 1. (D) A/B compartment distribution along chromosome 2. Cscores were calculated from Wt and del(attP-Rel5)d9lac E12.5 distal and proximal limb $\mathrm{Hi}-\mathrm{C}$ data. Compartment $\mathrm{A}$ is represented as positive values (red) and compartment B as negative values (blue). Gene density is shown in the bottom panel and the HoxD locus is indicated as a blue bar. (PDF 814 kb)

Additional file 2: Expression analysis around the HoxD associated transcripts. (A) WISH of lacZ mRNA in del(attP-Rel5)d9lac and del(113)d9lac E11.5 forelimbs (center) and whole embryos (left). The proximal domain is shown by an arrowhead. Hoxd10 and Hoxd11 WISH of E11.5 wild-type forelimbs are shown on the right for comparison of their expression domains. (B) WISH of Island3 eRNAs in Wt and del(attP-Rel5)d9lac E12.5 embryos where the antisense probe was used (left) showing the specificity on the digital region. On the right, a probe control shows the lack of staining with the Island3 sense probe. $(C, D)$ Volcano plots of all genes analyzed by RNA-seq in proximal (C) and distal (D) limb tissues comparing del(attPRel5)d9lac expression values to the control. All the genes located inside or in the vicinity of the 5'-TAD and the 3'-TAD are marked in red. Blue dots represent differentially expressed genes (absolute log2 fold change above 1.5 and adjusted $p$ value below 0.05 ) that are located outside these regions. Hoxd9 and Gm28793 (a short antisense mRNA) are significantly expressed due to their presence inside the Hoxd9/LacZ transgene. (E). RNA-seq-normalized read counts of wild-type versus del(attP-Re/5)d9lac distal limb tissue. The ten genes were selected according to the highest fold induction values obtained when wild-type distal and proximal tissues were compared. Statistical significance was assessed as adjusted $p$ values of the DESeq2 analysis. (PDF $14200 \mathrm{~kb})$

Additional file 3: $\mathrm{H} 3 \mathrm{~K} 27 \mathrm{me} 3$ distribution after disconnecting the HoxD from its 3'-TAD regulatory landscape. H3K27me3 ChIP profiles from proximal limb bud cells derived from either wild type, inv(attP-Itga6), or inv(Nsi-Itga6) specimens. $n$ indicates the number of replicates for each track. Below each mutant dataset, a comparison of mutant versus control 
is shown. A red asterisk in the control track indicates an artifactual signal at the position of island-3. (PDF $537 \mathrm{~kb}$ )

Additional file 4: (A) H3K27me3 coverage outside the HoxD locus in distal limb bud cells from either control (Wt) or del(attP-Rel5)d9lac specimens. A corresponding Hi-C map is shown on top spanning ca. $10 \mathrm{Mb}$ and centered around the HoxD cluster (blue box), with the related TAD structures (chr2:69600001-79440000). The flanking 5' and 3'-TAD TADs are indicated. H3K27me3 ChIP profiles in control cells show a global coverage outside the HoxD cluster precisely restricted to the 3'-TAD. In del(attP-Rel5)d9lac-mutant distal limb cells, the enrichment is much weaker. Below is the difference in the ChIP datasets comparing mutant versus control signals. The red asterisks point to an artifactual signal. (B) Quantification of H3K27me3 ChIP signal of wild type, del(attP-Rel5)d9lac, inv(attP-Itga6), and inv(Nsi-Itga6) in distal (left) or proximal (right) forelimb cells. The plotted values are computed from the regions depicted in (A) as dashed boxes. (PDF $2145 \mathrm{~kb}$ )

Additional file 5: $\mathrm{H} 3 \mathrm{~K} 27 \mathrm{me} 3$ signal over the HoxD cluster in the absence of the 5'-TAD. H3K27me3 ChIP profiles in distal (top two tracks) and proximal (bottom two tracks) limb bud cells, either in control $(W t)$ or in inv(Nsi-Itga6) mutant specimen. Below are shown the difference profiles. The increase of signal in Hoxd11 represents reads coming from the Hoxd11lac transgene included in the inversion allele (represented by a shaded gray box). (PDF $429 \mathrm{~kb}$ )

\section{Abbreviations}

3'-TAD: Telomeric TAD; 5'-TAD: Centromeric TAD; ChIP: Chromatin immunoprecipitation; eRNA: Enhancer RNA; IncRNA: Long non-coding RNA; PRC: Polycomb repressive complex; TAD: Topologically associating domain; WISH: Whole-mount in situ hybridization

\section{Acknowledgements}

We thank Leonardo Beccari, Aurélie Hintermann, Andréa Willemin, Jozsef Zákány, and other members of the Duboule laboratories for the insightful comments and discussion. We also thank Bénédicte Mascrez, Sandra Gitto, and Thi Hanh Nguyen Huynh for their help with mice breeding and genotyping, as well as Mylène Docquier, Brice Petit, and Christelle Barraclough from the Geneva Genomics platform (University of Geneva).

\section{Authors' contributions}

ERC and DD were responsible for the design of the experiments and manuscript writing. ERC and NYK were responsible for the bench work. LLD and AUA were responsible for the computing analysis. ERC, LLD, and DD were responsible for the analysis of the results. LLD, AUA and NYK were responsible for the manuscript correction. DD was responsible for the funding acquisition. All authors read and approved the final manuscript.

\section{Funding}

This work was supported by funds from the École Polytechnique Fédérale (EPFL, Lausanne), the University of Geneva, the Swiss National Research Fund (No. 310030B_138662), and the European Research Council grants SystemHox (No 232790) and RegulHox (No 588029) (to DD). The funding bodies had no role in the design of the study and collection, analysis and interpretation of data, and writing of the manuscript.

\section{Availability of data and materials}

The datasets generated and analyzed for this study are available in the GEO repository under accession number GSE129427 (https://www.ncbi.nlm.nih.gov/ geo/query/acc.cgi?acc=GSE129427) [74]. Publicly available data used in this paper can be found in GEO under the accession numbers GSE101715 (Hi-C) [34] and GSE101713 (4C-seq) [34]. The gtf file used for STAR and cufflinks, based on Ensembl version 94 annotations, is available on figshare [60].

\section{Ethics approval and consent to participate}

All experiments were performed in agreement with the Swiss Law on Animal Protection (LPA), under license No GE 81/14 (to DD).

\section{Consent for publication}

Not applicable.

\section{Competing interests}

The authors declare that they have no competing interests.

\section{Author details}

${ }^{1}$ Laboratory of Developmental Genomics, Department of Genetics and Evolution, University of Geneva, 1211 Geneva 4, Switzerland. ${ }^{2}$ School of Life Sciences, Federal Institute of Technology, Lausanne, 1015 Lausanne, Switzerland. ${ }^{3}$ Laboratory of Artificial and Natural Evolution, Department of Genetics and Evolution, University of Geneva, 1211 Geneva 4, Switzerland. ${ }^{4}$ Collège de France, 75005 Paris, France. ${ }^{5}$ Present Address: Laboratory for Developmental Genetics, RIKEN Center for Integrative Medical Sciences (IMS), 1-7-22 Suehiro-cho, Tsurumi-ku, Yokohoma, Kanagawa 230-0045, Japan.

Received: 12 April 2019 Accepted: 28 June 2019

Published online: 12 July 2019

\section{References}

1. Dekker J, Belmont AS, Guttman M, Leshyk VO, Lis JT, Lomvardas S, et al. The 4D nucleome project. Nature. 2017;549:219-26.

2. Davies JOJ, Oudelaar AM, Higgs DR, Hughes JR. How best to identify chromosomal interactions: a comparison of approaches. Nat Methods. 2017; 14:125-34.

3. Phillips-Cremins JE, Sauria ME, Sanyal A, Gerasimova TI, Lajoie BR, Bell JS, et al. Architectural protein subclasses shape 3D organization of genomes during lineage commitment. Cell. 2013;153:1281-95.

4. Laura Vian A, Pe A, Rao SS, Levens D, Lieberman Aiden E, Casellas R, et al. The energetics and physiological impact of cohesin extrusion. Cell. 2018; 173:1-14.

5. Rao SSP, Huntley MH, Durand NC, Stamenova EK, Bochkov ID, Robinson JT, et al. A 3D map of the human genome at kilobase resolution reveals principles of chromatin looping. Cell. 2014;159:1665-80.

6. Fudenberg G, Abdennur N, Imakaev M, Goloborodko A, Mirny LA. Emerging evidence of chromosome folding by loop extrusion. Cold Spring Harb Symp Quant Biol. 2017;82:45-55.

7. Dixon JR, Selvaraj S, Yue F, Kim A, Li Y, Shen Y, et al. Topological domains in mammalian genomes identified by analysis of chromatin interactions. Nature. 2012:485:376-80.

8. Nora EP, Lajoie BR, Schulz EG, Giorgetti L, Okamoto I, Servant N, et al. Spatial partitioning of the regulatory landscape of the $\mathrm{X}$-inactivation Centre. Nature. 2012;485:381-5.

9. Zhan Y, Mariani L, Barozzi I, Schulz EG, Bluthgen N, Stadler M, et al. Reciprocal insulation analysis of $\mathrm{Hi}-\mathrm{C}$ data shows that TADs represent a functionally but not structurally privileged scale in the hierarchical folding of chromosomes. Genome Res. 2017. https://doi.org/10.1101/gr.212803.116.

10. Spitz F, Gonzalez F, Duboule D. A global control region defines a chromosomal regulatory landscape containing the HoxD cluster. Cell. 2003; 113:405-17.

11. Harmston N, Ing-Simmons E, Tan G, Perry M, Merkenschlager M, Lenhard B. Topologically associating domains are ancient features that coincide with Metazoan clusters of extreme noncoding conservation. Nat Commun. 2017;8:441.

12. Ibn-Salem J, Muro EM, Andrade-Navarro MA. Co-regulation of paralog genes in the three-dimensional chromatin architecture. Nucleic Acids Res. 2017:45:81-91.

13. Schmitt AD, Hu M, Jung I, Xu Z, Qiu Y, Tan CL, et al. A compendium of chromatin contact maps reveals spatially active regions in the human genome. Cell Rep. 2016;17:2042-59.

14. Woltering JM, Noordermeer D, Leleu M, Duboule D. Conservation and divergence of regulatory strategies at Hox loci and the origin of tetrapod digits. PLoS Biol. 2014;12:e1001773.

15. Yakushiji-Kaminatsui N, Lopez-Delisle L, Bolt CC, Andrey G, Beccari L, Duboule D. Similarities and differences in the regulation of HoxD genes during chick and mouse limb development. PLoS Biol. 2018;16:e3000004.

16. Nora EP, Goloborodko A, Valton AL, Gibcus JH, Uebersohn A, Abdennur N, et al. Targeted degradation of CTCF decouples local insulation of chromosome domains from genomic compartmentalization. Cell. 2017;169: 930-944 e22.

17. Rao SSP, Huang S-C, Glenn St Hilaire B, Engreitz JM, Perez EM, Kieffer-Kwon K-R, et al. Cohesin loss eliminates all loop domains. Cell. 2017;171:305-320.e24 
18. Schwarzer W, Abdennur N, Goloborodko A, Pekowska A, Fudenberg G Loe-Mie $Y$, et al. Two independent modes of chromatin organization revealed by cohesin removal. Nature. 2017;551:51-6.

19. Soshnikova N, Montavon T, Leleu M, Galjart N, Duboule D. Functional analysis of CTCF during mammalian limb development. Dev Cell. 2010;19: 819-30.

20. Nuebler J, Fudenberg G, Imakaev M, Abdennur N, Mirny LA. Chromatin organization by an interplay of loop extrusion and compartmental segregation. Proc Natl Acad Sci. 2018;115:E6697-706.

21. Vieux-Rochas M, Fabre PJ, Leleu M, Duboule D, Noordermeer D. Clustering of mammalian Hox genes with other H3K27me3 targets within an active nuclear domain. Proc Natl Acad Sci U S A. 2015;112:4672-7.

22. Larson AG, Elnatan D, Keenen MM, Trnka MJ, Johnston JB, Burlingame AL, et al. Liquid droplet formation by HP1 a suggests a role for phase separation in heterochromatin. Nature. 2017;547:236-40.

23. Strom AR, Emelyanov AV, Mir M, Fyodorov DV, Darzacq X, Karpen GH. Phase separation drives heterochromatin domain formation. Nature. 2017;547:241-5.

24. Cruz-Molina S, Respuela P, Tebartz C, Kolovos P, Nikolic M, Fueyo R, et al. PRC2 facilitates the regulatory topology required for poised enhancer function during pluripotent stem cell differentiation. Cell Stem Cell. 2017;20: 689-705.e9.

25. Denholtz M, Bonora G, Chronis C, Splinter E, de Laat W, Ernst J, et al. Longrange chromatin contacts in embryonic stem cells reveal a role for pluripotency factors and polycomb proteins in genome organization. Cell Stem Cell. 2013;13:602-16.

26. Joshi O, Wang SY, Kuznetsova T, Atlasi Y, Peng T, Fabre PJ, et al. Dynamic reorganization of extremely long-range promoter-promoter interactions between two states of pluripotency. Cell Stem Cell. 2015;17:748-57.

27. Fabre PJ, Leleu M, Mormann BH, Lopez-Delisle L, Noordermeer D, Beccari L, et al. Large scale genomic reorganization of topological domains at the HoxD locus. Genome Biol. 2017;18:149.

28. Long HK, Prescott SL, Wysocka J. Ever-changing landscapes: transcriptional enhancers in development and evolution. Cell. 2016;167:1170-87.

29. Osterwalder M, Barozzi I, Tissières V, Fukuda-Yuzawa Y, Mannion BJ, Afzal SY, et al. Enhancer redundancy provides phenotypic robustness in mammalian development. Nature. 2018:554:239-43.

30. Spitz F. Gene regulation at a distance: from remote enhancers to 3D regulatory ensembles. Semin Cell Dev Biol. 2016;57:57-67.

31. Symmons O, Uslu W, Tsujimura T, Ruf S, Nassari S, Schwarzer W, et al. Functional and topological characteristics of mammalian regulatory domains. Genome Res. 2014;24:390-400.

32. Beccari L, Yakushiji-Kaminatsui N, Woltering JM, Necsulea A, Lonfat N, Rodriguez-Carballo E, et al. A role for HOX13 proteins in the regulatory switch between TADs at the HoxD locus. Genes Dev. 2016:30:1172-86.

33. Andrey G, Montavon T, Mascrez B, Gonzalez F, Noordermeer D, Leleu M, et al. A switch between topological domains underlies HoxD genes collinearity in mouse limbs. Science. 2013;340:1234167.

34. Rodriguez-Carballo E, Lopez-Delisle L, Zhan Y, Fabre PJ, Beccari L, El-Idrissi I, et al. The HoxD cluster is a dynamic and resilient TAD boundary controlling the segregation of antagonistic regulatory landscapes. Genes Dev. 2017;31: 2264-81.

35. Sheth R, Barozzi I, Langlais D, Osterwalder M, Nemec S, Carlson HL, et al. Distal limb patterning requires modulation of cis-regulatory activities by HOX13. Cell Rep. 2016;17:2913-26.

36. Serra F, Baù D, Goodstadt M, Castillo D, Filion GJ, Marti-Renom MA. Automatic analysis and 3D-modelling of $\mathrm{Hi}-\mathrm{C}$ data using TADbit reveals structural features of the fly chromatin colors. PLoS Comput Biol. 2017;13:e1005665.

37. Schep R, Necsulea A, Rodriguez-Carballo E, Guerreiro I, Andrey G, Nguyen Huynh $\mathrm{TH}$, et al. Control of Hoxd gene transcription in the mammary bud by hijacking a preexisting regulatory landscape. Proc Natl Acad Sci U A. 2016;113:E7720-9.

38. Tschopp P, Duboule D. A regulatory "landscape effect" over the HoxD cluster. Dev Biol. 2011;351:288-96.

39. Delpretti $\mathrm{S}$, Montavon $\mathrm{T}$, Leleu M, Joye $\mathrm{E}$, Tzika A, Milinkovitch M, et al. Multiple enhancers regulate Hoxd genes and the Hotdog LncRNA during cecum budding. Cell Rep. 2013;5:137-50.

40. Montavon T, Soshnikova N, Mascrez B, Joye E, Thevenet L, Splinter E, et al. A regulatory archipelago controls Hox genes transcription in digits. Cell. 2011; 147:1132-45.

41. Li H, Liefke R, Jiang J, Kurland JV, Tian W, Deng P, et al. Polycomb-like proteins link the PRC2 complex to CpG islands. Nature. 2017;549:287-91.
42. Riising EM, Comet I, Leblanc B, Wu X, Johansen JV, Helin K. Gene silencing triggers polycomb repressive complex 2 recruitment to $\mathrm{CpG}$ islands genome wide. Mol Cell. 2014;55:347-60.

43. Ku M, Koche RP, Rheinbay E, Mendenhall EM, Endoh M, Mikkelsen TS, et al. Genomewide analysis of PRC1 and PRC2 occupancy identifies two classes of bivalent domains. PLoS Genet. 2008;4:e1000242.

44. Tabin C, Wolpert $\mathrm{L}$. Rethinking the proximodistal axis of the vertebrate limb in the molecular era. Genes Dev. 2007;21:1433-42.

45. Kundu S, Ji F, Sunwoo H, Jain G, Lee JT, Sadreyev Rl, et al. Polycomb repressive complex 1 generates discrete compacted domains that change during differentiation. Mol Cell. 2017;65:432-446 e5.

46. Noordermeer D, Leleu M, Splinter E, Rougemont J, De Laat W, Duboule D. The dynamic architecture of Hox gene clusters. Science. 2011;334: 222-5.

47. Davis AP, Witte DP, Hsieh-Li HM, Potter SS, Capecchi MR. Absence of radius and ulna in mice lacking hoxa-11 and hoxd-11. Nature. 1995;375:791-5.

48. Fromental-Ramain C, Warot X, Messadecq N, LeMeur M, Dolle P, Chambon P. Hoxa-13 and Hoxd-13 play a crucial role in the patterning of the limb autopod. Development. 1996;122:2997-3011.

49. Kmita M, Tarchini B, Zakany J, Logan M, Tabin CJ, Duboule D. Early developmental arrest of mammalian limbs lacking HoxA/HoxD gene function. Nature. 2005;435:1113-6.

50. Zakany J, Fromental-Ramain C, Warot X, Duboule D. Regulation of number and size of digits by posterior Hox genes: a dose-dependent mechanism with potential evolutionary implications. Proc Natl Acad Sci U A. 1997;94: 13695-700.

51. Reinberg D, Vales LD. Chromatin domains rich in inheritance. Science. 2018; 361:33-4.

52. Oksuz O, Narendra V, Lee C-H, Descostes N, LeRoy G, Raviram R, et al. Capturing the onset of PRC2-mediated repressive domain formation. Mol Cell. 2018;70:1149-1162.e5.

53. Schorderet P, Lonfat N, Darbellay F, Tschopp P, Gitto S, Soshnikova N, et al. A genetic approach to the recruitment of PRC2 at the HoxD locus. PLoS Genet. 2013;9:e1003951.

54. Ho JWK, Jung YL, Liu T, Alver BH, Lee S, Ikegami K, et al. Comparative analysis of metazoan chromatin organization. Nature. 2014;512:449-52.

55. Le Dily F, Baù D, Pohl A, Vicent GP, Serra F, Soronellas D, et al. Distinct structural transitions of chromatin topological domains correlate with coordinated hormone-induced gene regulation. Genes Dev. 2014;28: 2151-62

56. Escobar T, Oksuz O, Descostes N, Bonasio R, Reinberg D. Precise redeposition of nucleosomes on repressive chromatin domains sustain epigenetic inheritance during DNA replication. bioRxiv. 2018. https://doi. org/10.1101/418707.

57. Reverón-Gómez N, González-Aguilera C, Stewart-Morgan KR, Petryk N, Flury $V$, Graziano S, et al. Accurate recycling of parental histones reproduces the histone modification landscape during DNA replication. Mol Cell. 2018;72: 239-249.e5

58. MUG VRE. MuG Virtual Research Environment. https://vre. multiscalegenomics.eu/home/. Accessed June 2019.

59. Zheng $\mathrm{X}$, Zheng Y. CscoreTool: fast Hi-C compartment analysis at high resolution. Bioinformatics. 2018;34:1568-70.

60. Lopez-Delisle L, Rodríguez-Carballo E, Duboule D. gtf file used in RodríguezCarballo et al. 2019. 2019. doi:https://doi.org/10.6084/m9.figshare.7775837.

61. Dobin A, Davis CA, Schlesinger F, Drenkow J, Zaleski C, Jha S, et al. STAR: ultrafast universal RNA-seq aligner. Bioinformatics. 2013;29:15-21.

62. Love Ml, Huber W, Anders S. Moderated estimation of fold change and dispersion for RNA-seq data with DESeq2. Genome Biol. 2014;15:550.

63. Montavon T, Le Garrec J-F, Kerszberg M, Duboule D. Modeling Hox gene regulation in digits: reverse collinearity and the molecular origin of thumbness. Genes Dev. 2008;22:346-59.

64. David FP, Delafontaine J, Carat S, Ross FJ, Lefebvre G, Jarosz Y, et al. HTSstation: a web application and open-access libraries for high-throughput sequencing data analysis. PLoS One. 2014:9:e85879.

65. Schmidl C, Rendeiro AF, Sheffield NC, Bock C. ChIPmentation: fast, robust, low-input ChIP-seq for histones and transcription factors. Nat Methods. 2015;12:963-5

66. Martin M. Cutadapt removes adapter sequences from high-throughput sequencing reads. EMBnet.journal. 2011;17. https://doi.org/10.14806/ej.17.1.200.

67. Langmead B, Salzberg SL. Fast gapped-read alignment with Bowtie 2. Nat Methods. 2012;9:357-9. 
68. Zhang Y, Liu T, Meyer CA, Eeckhoute J, Johnson DS, Bernstein BE, et al. Model-based analysis of ChIP-Seq (MACS). Genome Biol. 2008;9:R137.

69. Quinlan AR. BEDTools: the Swiss-Army tool for genome feature analysis. Curr Protoc Bioinforma. 2014:47:11 12 1-11 1234.

70. Broad Institute. Picard. http://broadinstitute.github.io/picard/. http:// broadinstitute.github.io/picard/. Accessed June 2019.

71. Ramirez F, Dundar F, Diehl S, Gruning BA, Manke T. deepTools: a flexible platform for exploring deep-sequencing data. Nucleic Acids Res. 2014;42 Web Server issue:W187-91.

72. Woltering JM, Vonk FJ, Muller H, Bardine N, Tuduce IL, de Bakker MA, et al. Axial patterning in snakes and caecilians: evidence for an alternative interpretation of the Hox code. Dev Biol. 2009;332:82-9.

73. Tarchini B, Duboule D. Control of Hoxd genes' collinearity during early limb development. Dev Cell. 2006;10:93-103.

74. Rodriguez-Carballo, E., Lopez-Delisle L, Yakushiji-Kaminatsui N, Ullate-Agote, A., Duboule D. Impact of genome architecture upon the functional activation and repression of Hox regulatory landscapes | bioRxiv. https://www.ncbi.nlm.nih. gov/geo/query/acc.cgi?acc=GSE129427. Accessed 3 Apr 2019.

\section{Publisher's Note}

Springer Nature remains neutral with regard to jurisdictional claims in published maps and institutional affiliations.

Ready to submit your research? Choose BMC and benefit from:

- fast, convenient online submission

- thorough peer review by experienced researchers in your field

- rapid publication on acceptance

- support for research data, including large and complex data types

- gold Open Access which fosters wider collaboration and increased citations

- maximum visibility for your research: over $100 \mathrm{M}$ website views per year

At BMC, research is always in progress.

Learn more biomedcentral.com/submissions 